\title{
Dynamical Systems with Controlled Singularities: Physically-Based Representation and Control-Oriented Modeling
}

\author{
J oseph Bentsman ${ }^{1}$ and Boris M. Miller²
}

\begin{abstract}
A bstract
A new class of systems: dynamical systems with controlled singularities, is introduced. This class refers to systems that admit impulsive control actions within their singular motion phases, the latter including changes in dimension, discontinuities in the state, and other nonsmooth types of motion. The motivation for this exort comes from various applications that admit sensing and/ or actuation ultra-fast in comparison to the natural system time scale, such as systems and mechanisms with impact-induced motion, power networks under faults, fast positioning devices, smart skins, and switching electronic circuits. The present work focuses on the class of dynamical systems with singular motions arising due to system inter action with controlled, or active, state constraints. The latter are assumed to be parametrizable by the elasticity-like coeф cient as follows: for the ...nite values of the coe cient the constraints are assumed to admit small violation dependent on the coec cient value, i.e., to display the elastic-type properties, wher eas in the limit as this coec cient tends to in..nity the constraint violation becomes inadmissible, i.e., the constraints become rigid. A physically well justi..ed representation of the above-mentioned class of systems is developed. This representation is found to require equations with the unbounded impulsive control signals in the right-hand-side (rhs) to describe the singular phase, making it not well suited for controller synthesis. Using a specially constructed topological map parametrized by the coeф cient of elasticity, with the latter tending to in...nity, the original representation is show $n$ to reduce in the limit to the simpli..ed one given by the nonlinear gener alized dixerential equations containing delta-functions in their rhs. This reduced, or limit, representation is shown to be a regularization of the original one due to the bounded ness of both the control signals and the coed cients of the delta-functions in the rhs of the system equations, and, consequently, to possess compatibility with the regular control design techniques. It is also shown to be capable of generating a unique isolated discontinuous system motion, i.e., to provide a tight and well-behaved description of the collision with rigid constraint. It is then demonstrated that the corresp onding paths gen erated by the original and the limit representations can be made ar bitrarily close to each other uniformly except, possibly, in the vicinities of the jump points by the appropriate choice of the value of the elasticity coec cient. This is shown to permit enforcing, for suф ciently large values of the elasticity coec cient, of the desired limit system behavior onto the original system by simply taking the control signals found through the limit representation, time-rescaling them, and inserting the resulting signals directly into the original system. These features show that limit system, in fact, provides a control-oriented representation of the class of systems considered. U sing this framework, two detailed examples of modeling in a di $₫$ cult to model class of systems - mechanical systems with control actions introduced through collisions with controlled nonstationary constraints, are given.
\end{abstract}

\section{K eywords}

$\mathrm{N}$ onsmooth analysis and synthesis, control of mechanical systems, singularities, systems with unilateral constraints, impacts, impulsive control, generalized solutions

\section{Int r oduct ion}

Systems that exhibit singularities in their behavior, such as discontinuities and nonsmoothness in system motion, jumps in dimension, lack of continuous dependence on the initial conditions, and nonuniqueness of solutions of the equations of motion are becoming more and more technologically important, and interest in their modeling and control, as well as development of new ultra-fast sensing and actuation capabilities during the singular phase is increasing ([14], [15], [22], [53], [58], [2], [3], [49]). For example, in mechanical systems collisions between the inter acting bodies cause the abrupt change in their velocities and thereby create the discretecont inuous behavior. Speci..c examples include robotic manipulators [42], [51],[53], vibro-impact mechanisms [1], walking (and, potentially, jumping) biped robots [22], and many others ( $c$. [14] with its extensive bibliography). M or eover, in the cases of juggling mechanical systems [13] and micro-electromechanical systems (MEMS) with micro-actuator arrays and thin-..Im impact microactuators ([6] [8], [25], and [31]) motion is induced only through organized impacts, hence it could be controlled only through these impacts. Such motion is especially important in MEMS since it is characterized by signi..cantly reduced friction and adhesion, known to be severe problems in this area. Ther efore, the exects of collision in modeling and control of these systems play a principal role and can not be neglected.

A nother large area of systems with nonsmooth motions is power systems, both at the generation and distribution levels. In these systems faults often have devastating exects. The topology of the power network is abruptly impacted by the fault, thereby inducing nonsmoothness in the network dynamics and a possibility of further fault propagation

1 Department of Mechanical and Industrial Engineering, U niversity of Illinois at U rbana-Champaign,1206 West Green Street, U rbana, IL 61801. E-mail: jbentsma@uiuc.edu, phone:(217)244-1076, fax:(217)244-6534.

${ }^{2}$ Institute for Information Transmission Problems, Russian Academy of Sciences, B. Karetny 19, 101447, M oscow, R ussia, e-mail: bmiller@iitp.ru 
[27], [54]. Other examples include space vehicles with impulsive propulsion [29], [30], economic systems with abrupt inputs in the form of sharp interest rate raises or cuts [18], [21], and quant um electronic systems [21].

Traditionally, the control exort in such systems has been either exerted during the nonsingular phase of the system motion or spent to induce the a priori rigidly speci..ed singular phase that does not admit any corrective actions within it. For example, at present, in the case of singularities induced by collision with constraints ([24], [15], [53], [22]), the latter are viewed as "passive impacters" which, during the phase of the engagement of the system with the constraint, enter passively into the determination of the post-impact system motion and do not substantially alter the motions induced by the interaction from impact to impact.

U pon detailed exam ination, however, of impact games such as soccer, ping-pong, tennis, and volleyball one can notice that the advanced player looks beyond the "novice" objective of simply changing the trajectory of the ball (and thereby introducing the trajectory nonsmoothness and velocity discontinuity) and attempts to gain advantage by pursu ing more sophisticated performance objectives, such as providing rotation of the ball and changing the velocity both in magnitude and direction, all during the short phase of interaction with the ball. The player in this setting can be viewed as generating a constraint and actively controlling its properties during the engagement phase. This player action gives rise to a new concept of active, or controlled, constraints, either naturally present or created through actuation, capable of radically changing the attainability set of the post-impact system state. The engagement phase of the system with such constraint can then be termed active singularity. These concepts, initially proposed by the authors in [3], [40], [41],[4], and [5], then naturally lead to the introduction of a new class of systems, dynamical systems with active, or controlled, singularities: a class of systems that admit control actions during the singular phases of their motion.

One immediately ..nds a number of applications where these concepts could be very bene..cial or even critically important. For example, in cutting systems, at the end of each pass the cutting torch re-enters the cutting path through the oval-shaped turning phase with the same velocity in the opposite direction to assure the uniformity of the cutting depth. This results both in the extra scrap metal and the increased cutting cycle duration. It, therefore, looks attractive to simply reverse the direction of the torch at the cutting path endpoint. However, to ensure that the velocity-dependent quantity of heat delivered by the torch provides the required uniformity of cutting in the vicinity of the endpoints, the position- and velocity-dependent impulsive control action needs to be exerted on the motion of the torch at the turning point to maintain the velocity of cutting but instantly change its direction. D ue to the impulsive forces required to execute such change, the dynamics of the turning phase will dixer from that of the regular one and will require ..ne structuring of the control signal for optimal execution of turning. Similar examples are positioning systems with precision/agility performance objectives, such as optical scanners, where it is necessary to apply magnetic/ electric impulses to the actuators during the moment of the direction change to attain optimal performance and/ or uniformity of exposure. A nother example is fault propagation stopping in the power networks via fast fault clearing which could be plausibly implemented through the impulsive control actions exerted in a power system during the fault.

The present work focuses on the class of dynamical systems that encompasses most of the applications described above, namely, systems with controlled, or active, unilateral constraints. The latter are assumed to be parametrized by the coed cient of elasticity: elastic, i.e. admitting a small constraint violation, for the ..nite coed cient value and rigid, i.e. forbidding the constraint violation, in the limit as the coec cient tends to in..nity. Naturally, bringing out and full utilization of the capabilities added by active constraints call for modeling framework that can i) incorporate the relevant phenomenological details, and ii) conveniently lend itself to the overall motion planning and controller synthesis for both regular and singular phases to enforce this motion. However, satisfying these, often con $\neq i c t i n g$, requirements for the class of systems considered turns out to be highly nontrivial. Indeed, examining available modeling techniques, for example, for mechanical systems subj ect to unilateral constraints with respect to the ..rst requirement, one ..nds that the conventional modeling of collisions, known to typically result in the velocity jumps in the system motion, makes use of either the so-called collision mapping or the restitution law, both of which give an expression for the velocity after the impact in terms of the velocity and position before the impact [1], [14], [15], [51]. The collision mapping, however, cannot analytically support description of the boundary of a time-varying and controlled, or active, constraint when the control forces during the phase of contact are impulsive.

The second requirement could be addressed by approximating the fast almost discontinuous motion of the actual system in the singular phase by the considerably simpler completely discontinuous, or limit, motion and, on the basis of the phenomenologically accurate model, generating the corresponding tight control-oriented models of limit dynamics with enough regularity to admit controller synthesis in both phases. Furthermore, the implications of applying the results obtained on the basis of such limit description to the actual system should be clari..ed, as well. Examining available techniques for modeling of limit behavior of systems with singular motions, one ..nds that this motion has been traditionally rigorously described by quasi-dixerential equations [49] and dixerential inclusions [50], [44], [46]. These representations, however, give system evolution not in terms of an isolated trajectory, but a set of trajectories, referred to as integral funnel. As a result, system motion is vaguely de..ned and its precise computation and control are problematic. In [45], [46], and [26] it is indicated that the extraction of an isolated system trajectory from the funnel can be accomplished through the use of dixerential equations with measure; however, the approach provided is 
not extendable to controlled singularities.

The present work addresses the issues raised above by making the following contributions to the existing literature:

a novel conceptual framework, that of systems with controlled singularities, is proposed and used to introduce the class of dynamical systems with controlled, or active, elastic unilateral constraints;

for this class of systems, a rigorous analytical framework is developed that resolves the aforementioned diф culties, speci..cally:

a system of dixer ential equations with unbounded impulsive control signals in the right-hand-side (rhs), further referred to as the original system, that consist ently accommodates controlled collisions, but is not well suited for motion planning and controller synthesis, is introduced;

a topological map - a one-to-one space-time transformation parametrized by the coet cient of elasticity is found that permits regularization of the original system;

applying this map to the original system, the corresponding limit representation is obtained for the in..nite value of the elasticity coet cient, and it is demonstrated that the corresponding solutions of the original and the limit representations can be made arbitrarily close to each other uniformly except, possibly, in the vicinities of the jump points by the appropriate setting of the value of the elasticity coed cient in the original system;

a new type of description of the impulsive action in the form of the controlled shift operator along the trajectories of the equation of fast dynamics in the singular phase is introduced and incorporated int o the system limit representation; this operat or can be viewed as active collision mapping, replacing the traditional one;

the limit representation is shown to have the desired modeling properties, namely, to uniquely describe isolated paths with discontinuities, have bounded rhs delta-function coeł cients and control signals, ret ain all the details of the original system dynamics, and, therefore, conveniently lend itself to simulation, system design, and cont rol law synthesis;

implementation of the control laws is shown to beaccomplished by simply substituting the time-rescaled bounded control signals found through the use of the limit representation into the actual physical system, resulting in the behavior of the latter close to that of the limit system for su $\$$ ciently large values of the elasticity coe cient and suф ciently accurate original system description;

using this framework, two detailed examples of modeling in a diф cult to model class of systems - mechanical systems with control actions introduced through collisions with controlled nonstationary constraints, are given.

The struct ure of the paper is as follows. Section II gives two motivating exam ples of systems that do not lend themselves to obtaining good models using the existing theory. The general model of a system with active constraints, the spacetime transformation, and the multi-scale system description are introduced in Section III. The limit representations of systems with one singularity and multiple singularities are derived in Sections IV and V, respectively. Section VI takes the examples of Section II and shows in detail how to use the framework developed in Sections III - V to obtain their limit representation. F inally, Section VII draws the conclusions. Appendix provides the proofs of some technical results.

\section{Mot ivat ing exampl es}

In this section two examples of a racket interacting with a ball are introduced. Both examples do not admit tight description of their dynamics using the available modeling techniques. The dixer ence between the examples is that in the ..rst one the velocity of the boundary surface during an impact does not change abruptly, whereas in the second one the rate of the velocity change during an impact is of the same order as that of the velocity of a bouncing ball. The examples, therefore, represent systems with passive and active singularities, respectively. The constraints are assumed to be parametrized by the coec cient of elasticity ${ }^{1}$, elastic and rigid for ..nite and in..nite ${ }^{1}$, respectively. The elasticity is permitted to be non-ideal, in general, with the non-ideality characterized by the restitution coec cient $k$.

\section{A. Example 1: System with a Passive Singul arity: Modeling of a Ball Colliding wit h Stat ionary and Moving Racket s}

Consider a ball of mass $\mathrm{m}$ with a two-coor dinate motion colliding with an elastic obstacle of mass $\mathrm{M}$, such as a racket, that has a single coordinate motion. Let $\left(x_{p}^{1} ; x_{p}^{2}\right)$ be the horizontal and the vertical coordinates of the moving ball, respectively, $\left(x_{v}^{1} ; x_{v}^{2}\right)$ be the corresponding velocities, and $\left(X_{p} ; X_{v}\right)$ be the coordinate and velocity of the obstacle surface in the vertical direction.

Then, the system has the state vector $z=\left(x_{p}^{1} ; x_{p}^{2} ; x_{p} ; x_{v}^{1} ; x_{v}^{2} ; x_{v}\right)$. The constraint which de. nes the free motion area is given by the relation $G(z)=X_{p} i \quad x_{p}^{2} \quad 0$ : The impact occurs at $t=i$ when $X_{p}(i)$ i $x_{p}^{2}(i)=0$.

The continuous motion description in the presence of the ..nite elasticity ${ }^{1}<1$ is standard. The goal at hand, however, is to obtain the velocity jump representation corresponding to the limit motion in the case of 1 ! 1 . In the absence of dry friction, this problem can be solved through the use of the mechanical conservation laws. In the presence of the dry friction, however, this problem becomes much more complicated, since both the elastic and the friction forces are of the impulsive type and their structures depend on the current abruptly changing vertical component of velocity. Figures $1-3$ show the typical cases arising in this problem. 


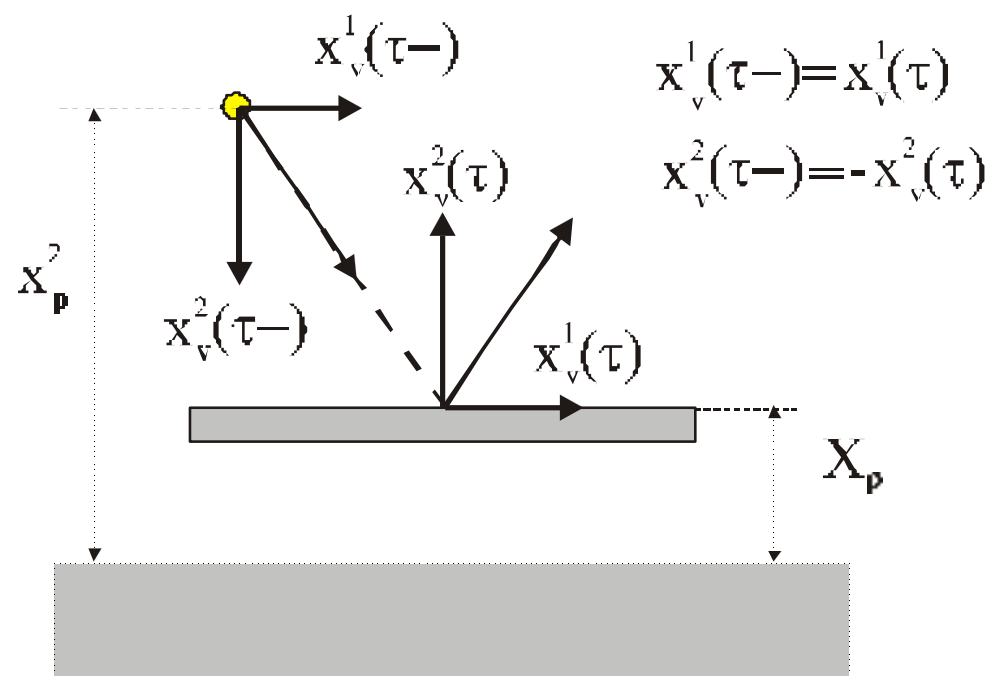

Fig. 1. I mpact with stationary ideally-elactic surface, restitution coeł cient $k=1$, no dry friction.

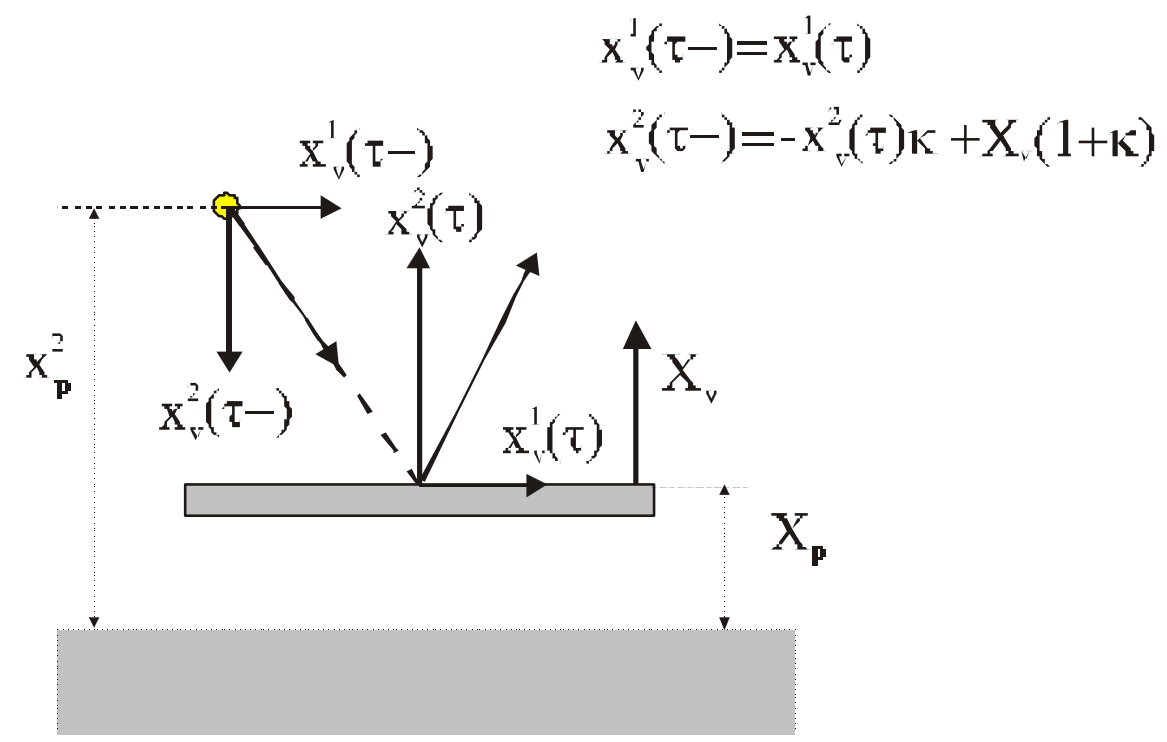

Fig. 2. Impact with moving non-ideally elastic surface, restitution coe $\$$ cient $(0<k<1)$, and no dry friction.

In the ..rst case, with the presence of the ideal elasticity, the stationary racket, and the absence of dry friction, the velocity after the impact can be calculated as shown in Fig.1. For the sake of simplicity it is assumed that $m_{i} \quad M$ (the general case will be considered below in the Section VI).

The presence of the non-ideal elasticity and the movement of the racket modify the vertical velocity component of the ball as shown in Fig. 2

The impact in the presence of dry friction is shown in Fig. 3.

In the latter case it is not possible to calculate the horizontal velocity component of the ball solely on the basis of the conservation laws without taking into account the dynamics of the contact. Indeed, here the component $x_{v}^{1}(i)$ depends not only on all the velocities prior to the impact, but also on the pro..le of the elastic reaction force. Ther efore, if one would like to obtain a tight description of the real motion, the impulsive nature of the impact has to be brought out in full detail. Otherwise, the only possibility would be to provide a rather loose system description through the use of dixerential inclusions like $x_{v}^{1}(i)<x_{v}^{1}(i i)$ or their analogs.

B. Example 2: System with an Active Singul arity: Model ing of a Ball Colliding with a Moving Racket that Rotat es During an Impact

Let us now consider the example motivated by the impact games, like ping-pong, where the player rotates the racket surface during the phase of contact of a racket with a ball. In this case, the additional force component arises due to the 


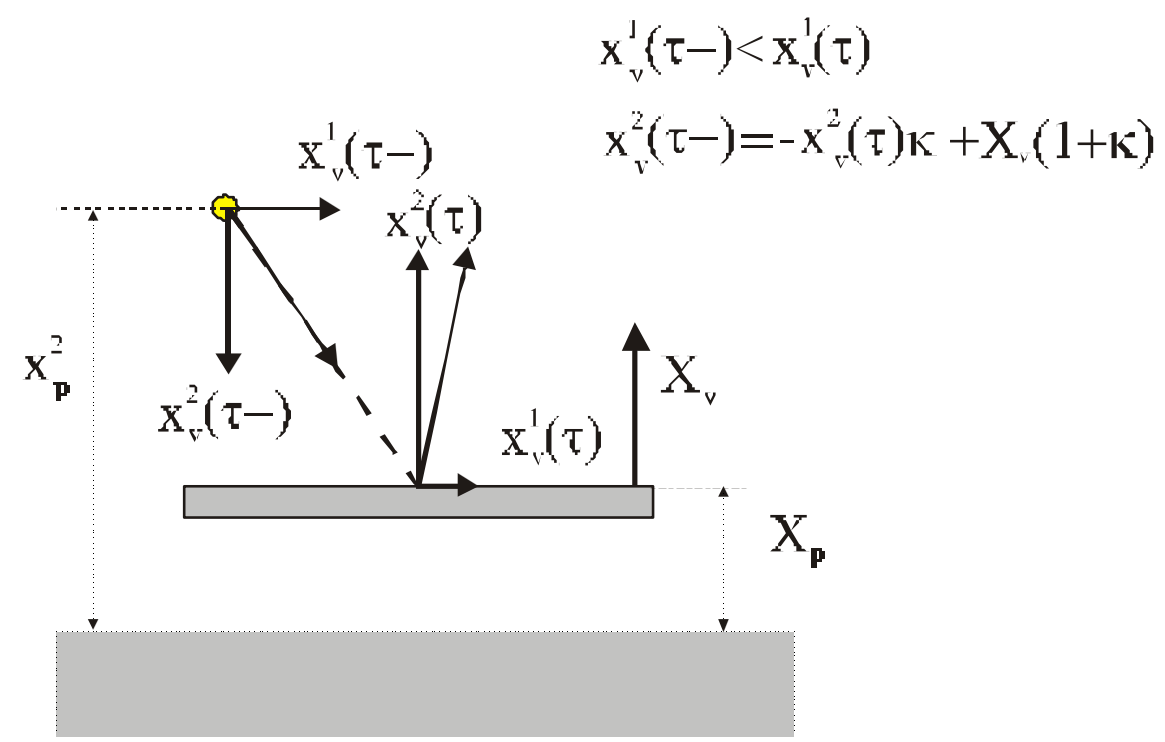

Fig. 3. Impact with moving non-ideally elastic surface, restitution coeç cient $(0<k<1)$, and dry friction present.

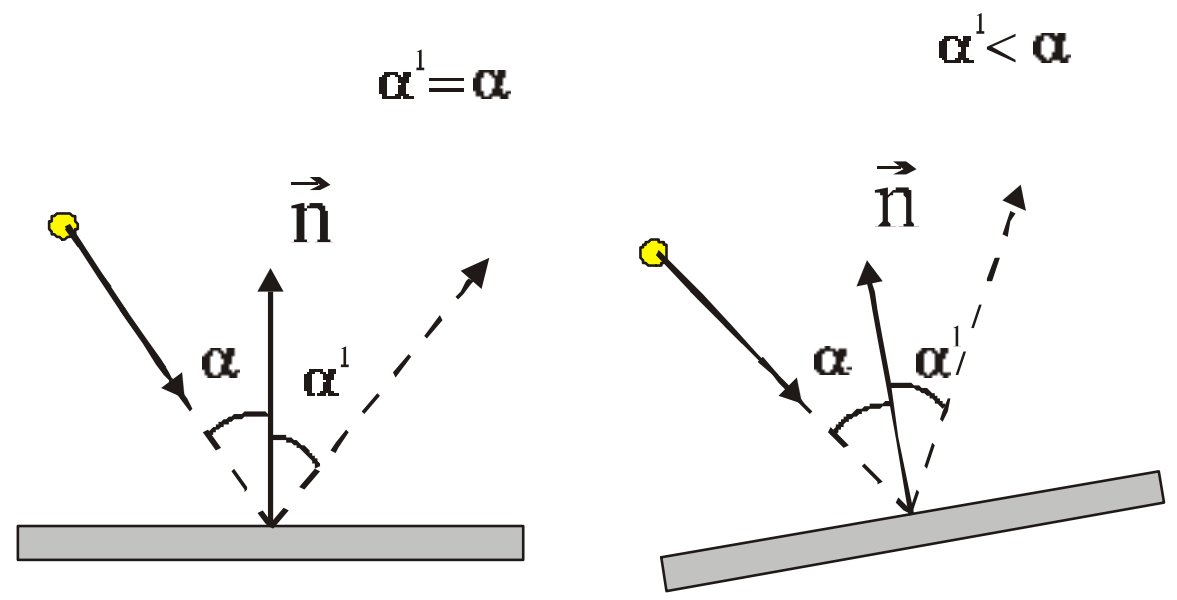

Fig. 4. I mpact with fast rotating racket.

change of the racket surface orientation, as shown in Fig. 4, where $\AA^{\circledR}$ and $\AA^{0}$ denote the pre- and post-impact rełection angles of the ball with respect to normals to the racket surface.

Such racket rotation during the very fast contact phase is frequently demonstrated by the highly skilled ping-pong players. If one tries to derive a collision map through the use of the mechanical conservation laws, one easily comes to the conclusion that these laws are insuф cient for describing the impulsive reaction of the rotating elastic surface, since both the orientation and the value of the reaction force abruptly change during the impact phase. This situation presents an even greater modeling challenge than that in the previous example due to the abrupt change of several position coordinates (like the normal to the racket surface) during contact. The typical behavior of $x_{p}(\$) ; x_{v}(\Phi)$ is shown in Fig. 5

The remainder of the present work develops the analytical setting capable of addressing the challenge posed by the examples described in this section.

\section{Dynamical Syst ems with Controlled Singul arities: the Physically-Based Model and the Mult i-Scal e Repr esent at ion.}

In order to approach the problems posed by the examples in Section II, one ..rst needs to focus on the phase of the constraint engagement. In the collision of rigid bodies, the constraints are not perfectly rigid and undergo small violation, giving rise to the contact forces. Therefore, in reality, there is a very fast, but continuous, phase of motion that looks discontinuous only with respect to the velocities in the natural time scale. If one considers this phase in the enlarged spatio-temporal scale generated via some space-time transformation, one can obtain a more detailed description of the collision. In [33], [34], and [42] this ap proach is used to derive the equations of collision mapping for robotic manipulator; 


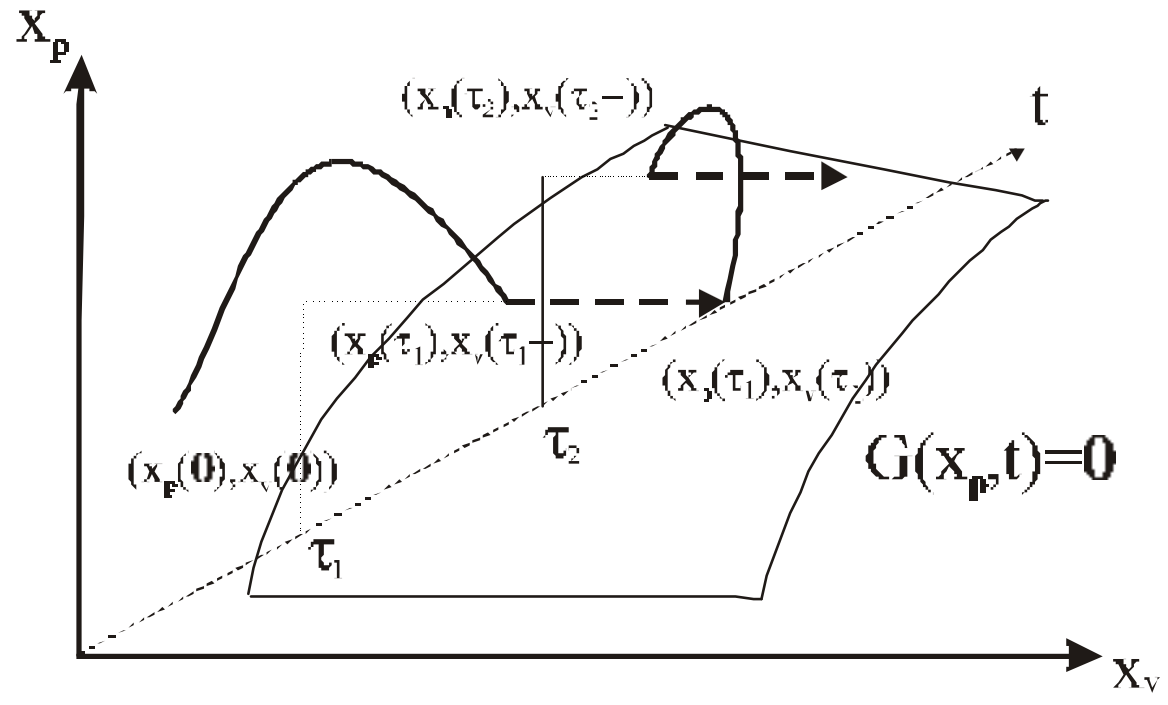

Fig. 5. Evolution of the limit system.

however, the description of the discontinuous system behavior is not rigorously obtained.

Further on, unlike the "passive constraints" case considered in [33], [34], and [42], in the case of the act ive constraints the impulsive control action should be admitted during the contact and properly represented in the system model. It looks reasonable to explore whether the formalism of the impulsive control theory (cf. [55], [56], [9], [10], [11], [12], [36], [37], [48] and many others) could be utilized for this purpose; however, one ..nds it to be not directly applicable to the case of constrained dynamical systems. This is due to the fact that the impulsive control setting considers large short duration cont rol inputs to be external to the system, whereas large short duration cont act forces arising during collision are inherently present in the constrained system itself and should be considered as the internal ones.

Based on these considerations, a physically motivated approach to generating the descriptions of systems with the contact impulsive forces is proposed with the following features:

2 the contact force is considered to be the result of a small violation of a constraint that starts taking place as soon as the system hits the boundary of a constraint;

2 this force resists the penetration of the system into the domain, inhibited by the constraint, and causes a very fast (almost instantaneous) change of the sign of normal velocity component to the opposite one;

2 the controlled motion in the inhibited domain is admitted and described by the nonstandard controlled singularly perturbed dixerential equation with in..nitely growing right-hand-side, such that the solution of this equation behaves in the limit as a stepwise function with respect to the components of the generalized velocity.

A. Nat ural and Singul ar Motion Phases for Syst ems with Cont rolled Singularities: the Gener al Syst em Model

Let in a system with elastic constraints the elasticity be parametrized by some coed cient ${ }^{1}$. Let the constraints admit a system motion, al beit inhibited, within the area occupied by them for ..nite ${ }^{1}$ and become rigid for ${ }^{1}$ ! 1 . Further on, consider decomposition of the entire system motion into two phases, the one corresponding to the motion in the area free of constraints and the second one describing the motion in the area inhibited by constraints, referred to as the natural and the singular phases, respectively.

Let the controlled dynamical system be described by the set of variables $x_{p}(t) 2 R^{n} ; x_{v}(t) 2 R^{n}$; where vector $x_{p}$ is referred to as the set of generalized coordinates and $x_{v}$ as the set of generalized velocities. Suppose that there is some constraint given in the form of the inequality

$$
\mathrm{G}\left(\mathrm{x}_{\mathrm{p}}(\mathrm{t}) ; \mathrm{t}\right) \quad 0
$$

were $G(x ; t)$ is the continuous and sul ciently smooth function. Let the system admit the two modes of motion, an unconstrained, or motion in the constraint-free area, and an "inhibited", or motion in the area occupied by the constraint, further referred to as the natural and the singular motion phases, respectively. 


\section{A.1 Motion in the Nat ural Phase}

In the domain $f\left(x_{p} ; t\right): G\left(x_{p} ; t\right) \quad 0 g$ the system of dixerential equations for $f x_{p} ; x_{v} g$ has the form

$$
\begin{aligned}
& \underline{x}_{p}(t)=F_{p}^{r}\left(x_{p}(t) ; x_{v}(t) ; u(t) ; t\right) ; \\
& \underline{x}_{v}(t)=F_{v}^{r}\left(x_{p}(t) ; x_{v}(t) ; u(t) ; t\right) ;
\end{aligned}
$$

where $u(t) 2 U 1 / 2 R^{m}$ is some control, $U$ is a compact set, and $F_{v}^{r}\left(x_{p} ; x_{v} ; u ; t\right)$ and $F_{p}^{r}\left(x_{p} ; x_{v} ; u ; t\right)$ have the standard properties of continuity and smoothness suф cient for existence and uniqueness of the solution of system (2) for a given measurable control $u\left(\phi\right.$ and arbitrary initial conditions $x_{p}(0) ; x_{v}(0)$. For example, they could be assumed to be continuous with respect to all variables and smooth with respect to $\left(x_{p} ; x_{v}\right)$ :

\section{A.2 Motion in the Singul ar Phase}

In the domain $f\left(x_{p} ; t\right): G\left(x_{p} ; t\right)>0 g$ the system of dixerential equations for $f x_{p} ; x_{v} g$ has the form

$$
\begin{aligned}
& \underline{x}_{p}(t)=F_{p}^{r}\left(x_{p}(t) ; x_{v}(t) ; u(t) ; t\right) ; \\
& \underline{x}_{v}(t)={ }^{1} F_{v}^{s}\left(x_{p}(t) ; x_{v}(t) ; w\left(t ;{ }^{1}\right) ; t^{1}{ }^{1}\right)+F_{v}^{r}\left(x_{p}(t) ; x_{v}(t) ; u(t) ; t\right) ;
\end{aligned}
$$

where ${ }^{1} \mathrm{~F}_{\mathrm{v}}^{\mathrm{S}}\left(\mathrm{x}_{\mathrm{p}} ; \mathrm{x}_{\mathrm{v}} ; \mathrm{w}\left(\mathrm{t} ;{ }^{1}\right) ; \mathrm{t} ;{ }^{1}\right)$ describes an additional controlled contact force, with $w\left(t ;{ }^{1}\right) 2 \mathrm{~W}$ being the external contact force control signal. A s before, this contact for ce is considered to arise due to the constraints violation. Function $\mathrm{F}_{\mathrm{v}}^{\mathrm{S}}\left(\mathrm{x}_{\mathrm{p}} ; \mathrm{x}_{\mathrm{v}} ; \mathrm{w} ; \mathrm{t}^{1}{ }^{1}\right)$ is supposed to be continuous and smooth in the area $\mathrm{G}\left(\mathrm{x}_{\mathrm{p}} ; \dot{\Sigma}\right), 0$; and satisfy the constraints

$$
F_{v}^{S}\left(x_{p} ; x_{v} ; w ; t^{1}\right)=0 ; \quad \text { if } G\left(x_{p} ; t\right)=0 ; \quad \frac{d \bar{d}}{d t}{ }_{F_{p}^{r}}^{-} \quad G\left(x_{p} ; t\right)=0 \text { : }
$$

A ssume that for any given $0 \quad 1<1$ the joint system (2),(3) has the unique solution for any given measurable controls $\mathrm{u}(\phi ; \mathrm{w}(\phi)$ : The objective is to determine the behavior of the joint system for 1 " 1 ; and to ..nd out if there exists the appropriate limit for its solution. If the limit exists, one can treat it as the generalized solution of a dynamical system with unilateral constraints, which would then bedescribed by the limiting form of the joint system - a dixerential equation with delta functions in the rhs, or, more generally, with measure.

The equations (2) and (3) together with the constraint (1) will be referred to as the original system.

Remark 1: T he principal feature of the systems considered is that their generalized coordinates are continuous while their velocities admit the jumps. At the same time, the generalized coor dinates are exactly the ones responsible for the appearance of the contact forces. Therefore, in order to properly describe system behavior one needs to carry out a more involved analysis in the vicinity of the constraint violation points. It should be noted that the standard machinery of the singular perturbations analysis does not provide the possibility for clearly bringing out the nat ure of the impacts. The typical results that could be obtained with the aid of the singular perturbations analysis give the solutions that do not depend on the state preceding the impact phase. This, however, can not bethe case in impact dynamics. Therefore, to describe the genuine impulsive nature of the impact, one needs to introduce the multi-scale motion representation obtained through "opening up" of a singularity, i.e. modeling of its ..ne structure, which can be accomplished through the use of the space-time transformation in the vicinity of the singularity point.

\section{B. Space-Time Transformation at the Singul ar it y Point and the Multi-Scale Syst em Descript ion}

Let the system start from the initial condition $x_{p}(0) ; x_{v}(0)$ such that $G\left(x_{p}(0) ; 0\right)<0$ and $i$ be the ..rst point where the system engages the constraint, so that

$$
\left.\mathrm{G}\left(\mathrm{x}_{\mathrm{p}}(\dot{i}) ; \dot{i}\right)=0 ; \quad \frac{\mathrm{d} \overline{-}}{\mathrm{dt}}{ }_{\mathrm{F}_{\mathrm{p}}^{r}}^{-} \mathrm{G}\left(\mathrm{x}_{\mathrm{p}}(\dot{i}) ; \dot{i}\right)\right)>0 ;
$$

and control $w\left(t ;{ }^{1}\right)$ has a form

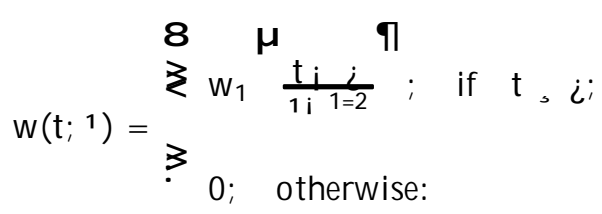


Therefore, for ..nite value of ${ }^{1}$ there exists a non-zer o time interval of the constraints violation. Introduce the following space time transformation of the coordinates and the independent variable for $\mathrm{s}>0$ :

$$
\begin{aligned}
& y_{p}^{1}(s)=x_{p}(i)+{ }^{11=2}\left[x_{p}(i+1 i 1=2 s) ; x_{p}(i)\right] ; \\
& y_{v}^{1}(s)=x_{v}\left(i+{ }^{1 i 1=2} s\right) ; \\
& t=i+{ }^{1 i}{ }^{1=2} s:
\end{aligned}
$$

Then the new variables $f y_{p}^{1}(s) ; y_{v}^{1}(s) g$ satisfy the equations

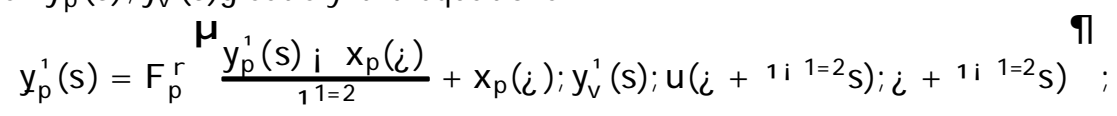

$$
\begin{aligned}
& \underline{y}_{v}^{1}(s)=11=2 F_{v}^{s} \frac{y_{p}^{1}(s) i x_{p}(i)}{11=2}+x_{p}(i) ; y_{v}^{1}(s) ; w_{1}(s) ; i+1 i 1=2 s^{1}{ }^{1}+ \\
& \left.1 \text { i } 1=2 \mathrm{~F}_{\mathrm{v}}^{\mathrm{r}} \frac{\mathrm{y}_{\mathrm{p}}^{1}(\mathrm{~s}) i \mathrm{x}_{\mathrm{p}}(i)}{11=2}+\mathrm{x}_{\mathrm{p}}(i) ; \mathrm{y}_{\mathrm{v}}^{1}(\mathrm{~s}) ; u(i+1 ; 1=2 \mathrm{~s}) ; i+1 ; 1=2 \mathrm{~s}\right) \text {; } \\
& y_{p}^{1}(0)=x_{p}(i) ; \quad y_{v}^{1}(0)=x_{v}(i i):
\end{aligned}
$$

The system of equations (2) for the nonsingular phase, the coordinate map (6), and the system of equations (7) for the singular phase will be jointly referred to as the multi-scale motion representation of the original system. This term arises due to the decomposition of the original system equations (2) and (3) that contain mixed scales into subsystems separately describing the slow regular and the fast singular phases.

IV. Dynamical Systems with Cont roll ed Singul ar it ies: Limit Representation of a Singl e J ump.

Next theorem describes the limit behavior of singularly perturbed system (7) as ${ }^{1}$ " 1 : T he theorem and its corollary demonstrate that the velocity jumps can be represented by means of the shift-operator along the paths of some limit system of dixerential equations. The theorem also shows how to incorporate control into the singular motion phase and thereby creates the bridge between impact mechanics and the impulsive control theory.

A. Cal culation of the jump of the gener alized vel ocity

A ssumption 1: Suppose that $\mathrm{F}_{\mathrm{v}}^{\mathrm{s}}$ satis..es the Lipschitz condition in the following form: there exists $L>0 ;{ }^{1}{ }_{0}>0$ such that for any $\left(\mathrm{x}_{\mathrm{p}} ; \mathrm{x}_{\mathrm{p}}^{0} ; \mathrm{x}_{\mathrm{v}} ; \mathrm{x}_{\mathrm{v}}^{0}\right) ; \mathrm{t} 2[0 ; \mathrm{T}] ; \mathrm{w} 2 \mathrm{~W}$; and ${ }^{1},{ }^{1}{ }_{0}$

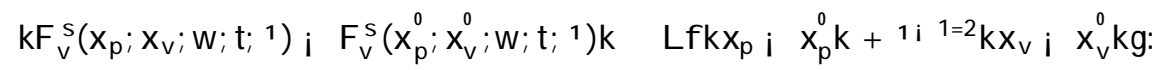

Theorem 1: Assume that:

1. 1) for any $\left(x_{p} ; i\right)$ such that $G\left(x_{p} ; i\right)=0$ there exists

$$
\begin{aligned}
& \left.\lim _{1,1} F_{p}^{r} \frac{\mu_{p} i x_{p}}{11=2}+x_{p} ; y_{v} ; u(i+1 i 1=2 s) ; i+1 ; 1=2 s\right)= \\
& =F_{p}\left(y_{p} ; \Psi_{v} ; u(\Sigma) ; x_{p} ; \dot{L}\right) ; \\
& \lim _{1+1}{ }^{11=2} F^{s} \frac{{ }^{\mu} p_{p} i x_{p}}{11=2}+x_{p} ; y_{v} ; w_{1}(s) ; i+{ }^{1 i}{ }^{1=2} s^{1}{ }^{1 "}= \\
& F_{v}\left(y_{p} ; y_{v} ; s ; w_{1}(s) ; x_{p} ; \dot{c}\right) ;
\end{aligned}
$$

where convergence is uniform in any bounded vicinity of $\left(y_{p} ; y_{v} ; s\right)$;

2) the limit system of dixerential equations, i.e.

$$
\begin{aligned}
& y_{p}(s)=F_{p}\left(y_{p}(s) ; y_{v}(s) ; w_{1}(s) ; x_{p}(i) ; i\right) ; \\
& y_{v}(s)=F_{v}\left(y_{p}(s) ; y_{v}(s) ; s ; w_{1}(s) ; x_{p}(i) ; i\right) ; \\
& y_{p}(0)=x_{p}(i) ; \quad y_{v}(0)=x_{v}(i i):
\end{aligned}
$$


has the unique solution on some interval $\left[0 ; \mathrm{s}^{\not}+\right.$ "]; where " $>0$ and

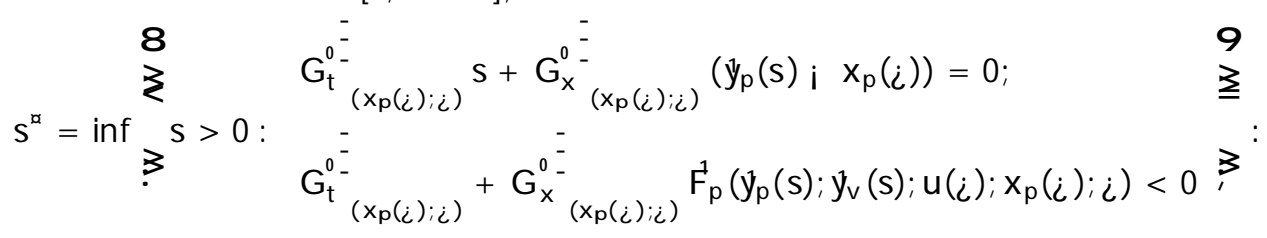

Then, if ${ }^{1}$ ! 1 ;

$$
\left(y_{p}^{1}(s) ; y_{v}^{1}(s)\right) !\left(y_{p}(s) ; y_{v}(s)\right) \text { uniformly on }\left[0 ; s^{x}+"\right] \text {; }
$$

and for all su ciently large $^{1}$ there exists

$$
\begin{aligned}
& \mathrm{G}\left(\mathrm{X}_{\mathrm{p}}\left(i+1 \mathrm{i}^{1=2} \mathrm{~s}\right) ; i+{ }^{1} \mathrm{i}^{1=2} \mathrm{~S}\right)=0 \text {; } \\
& S_{1}^{\alpha}=\inf S>0: G_{t}^{0} j_{\left(x_{p}(i+1 ; 1=2 s) ; i+1 ; 1=2 s\right)}+ \\
& \left.+G_{x}^{0} j_{\left(x_{p}(i+1 i 1=2 s) ; i+1 i 1=2 s\right)} F_{p}^{r}{ }^{i} x_{p}(i+1 i 1=2 s) ; x_{v}(i+1 i 1=2 s) ; u(i+1 i 1=2 s) ; i+1 i 1=2 s\right)<0 ;
\end{aligned}
$$

such that

$$
s_{1}^{\alpha} ! s^{\alpha}:
$$

Proof: The continuity of the ordinary dixerential equation solution with respect to the parameters (cf. [19] $p$. 71 or [50] Th.7 §1) implies that $\left(y_{p}^{1}(s) ; y_{v}^{1}(s)\right)$ converges to! $\left(y_{p}(s) ; y_{v}(s)\right)$ uniformly on $\left[0 ; s^{x}+\right.$ "]: This follows from Lipschitz condition (8) and assumptionl of Theorem 1.

To prove the second part of Theorem let us de..ne

$$
f^{0}(s)=G_{x}^{0}\left(x_{p}(i) ; i\right)\left(y_{p}(s) i x_{p}(i)\right)+G_{t}^{0}\left(x_{p}(i) ; i\right) s ;
$$

and

$$
f^{1}(s)=G_{x}^{0}\left(x_{p}(i+1 i 1=2 s) ; i+{ }^{1 i 1}{ }^{1=2} s\right)\left(y_{p}^{1}(s) i x_{p}(i)\right)+G_{t}^{0}\left(x_{p}\left(i+{ }^{1 i 1} 1=2 s\right) ; i+{ }^{1 i 1}{ }^{1=2} s\right) s:
$$

Then

$$
f_{-}^{0}(s)=G_{x}^{0}\left(x_{p}(i) ; i\right) \mathbb{Z}_{p}(s)+G_{t}^{0}\left(x_{p}(i) ; i\right) ;
$$

and

$$
f_{-}^{1}(s)=G_{x}^{0}\left(x_{p}(i+1 i 1=2 s) ; i+1^{1 i 1=2} s\right) y_{p}^{1}(s)+G_{t}^{0}\left(x_{p}(i+1 i 1=2 s) ; i+1^{1 i 1=2} s\right)+O\left({ }^{1} i^{1=2}\right):
$$

The proof follows from uniform convergence of $\left(\mathrm{y}_{p}^{1}(\mathrm{~s}) ; \mathrm{Y}_{p}^{1}(\mathrm{~s})\right)$ to $\left(\mathrm{y}_{\mathrm{p}}(\mathrm{s}) ; \mathbb{y}_{\mathrm{p}}(\mathrm{s})\right)$ and the lemma below, the proof of which is given in A ppendix.

Lemma 1: For any continuous and smooth function $f(\phi)$ de..ne an exit time

$$
i^{ \pm}(\mathrm{f})=\begin{aligned}
& 8 \\
& <\operatorname{inffs}>0: \mathrm{f}(\mathrm{s}) \quad \pm \mathrm{tg} ; \\
& 1 ; \quad \text { if the set is empty: }
\end{aligned}
$$

Let for some function $f^{0}\left(\Phi\right.$ such that $f^{0}(0)>0$ the exit time $i^{0}\left(f^{0}\right)<1$ and $f^{0}$ have the negative derivative at $t=i^{0}\left(f^{0}\right)$ : Then $i^{0}(f)$ is continuous at $f^{0}(\phi$ with respect to the topology of uniform convergence. In other words, if $f^{n}(s)$ ! $f^{0}(s)$ uniformly on some interval $\left[0 ; i^{0}\left(f^{0}\right)+"\right]$; " $>0$; then

By lemma there exists $\dot{\iota}^{0}\left(f^{1}\right) ! \quad i^{0}\left(f^{0}\right)=s^{\not}$ : Then

$$
i^{0}\left(f^{n}\right) ! \quad i^{0}\left(f^{0}\right):
$$

$$
G\left(x_{p}\left(i+1 i^{1=2} s\right) ; i+{ }^{1} i^{1=2} s\right) ; G\left(x_{p}(i) ; i\right)=
$$

$$
=1 i 1=2^{h} G_{x}^{0}\left(x_{p}(i+1 i 1=2 s) ; i+1 i 1=2 s\right)\left(y_{p}^{1}(s) i x_{p}\right)+G_{t}^{0}\left(x_{p}(i+1 i 1=2 s) ; i+1 i 1=2 s\right) s+O\left({ }^{1} i 1\right) ;
$$


where $\frac{\left.\mathrm{O}^{1} \mathrm{i}^{1}\right)}{1 \mathrm{i}^{1=2}}$ ! 0 ; if ${ }^{1}$ ! 1 uniformly in $\mathrm{s} 2\left[0 ; \mathrm{s}^{\infty}+\right.$ "]: Since $\mathrm{G}\left(\mathrm{x}_{\mathrm{p}}(\dot{\Sigma}) ; \dot{\Sigma}\right)=0$; we have

$$
{ }^{1}{ }^{1=2} G\left(x_{p}\left(i+1 i^{1=2} s\right) ; i+1 i^{1=2} s\right)=f^{1}(s)+\frac{O\left(1 i^{1}\right)}{1 i^{1=2}}
$$

D ue to the negativity of the derivative of $f^{0}$ at $i^{0}\left(f^{0}\right)$ there exists \pm such that for any $\pm 2(0 ; \pm)$

$$
i^{ \pm}\left(f^{0}\right)<i^{i \pm}\left(f^{0}\right) \quad s^{\alpha}+":
$$

Then, the uniform convergence of $\mathrm{f}^{1}$ to $\mathrm{f}^{0}$ for chosen \pm and su $\phi$ ciently large ${ }^{1}>^{1}{ }_{1}\left(\uplus_{1}\right.$ yields the inequalities

$$
f^{1}\left(i^{ \pm}\left(f^{0}\right)\right), \quad \frac{ \pm}{2} ; \quad f^{1}\left(i^{i}\left(f^{0}\right)\right) \quad \text { i } \frac{ \pm}{2}
$$

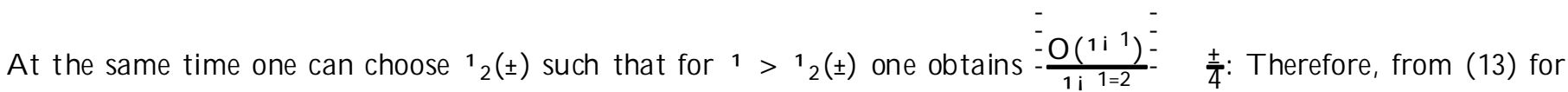
suф ciently large ${ }^{1}$ one has

$$
{ }^{1}{ }^{1=2} G\left(x_{p}\left(i+1 \text { i } 1=2{ }^{ \pm}\left(f^{0}\right)\right) ; i+1 \text { i } 1=2{ }^{ \pm}\left(f^{0}\right)\right)>\frac{ \pm}{4} ; \quad 11=2 G\left(x_{p}\left(i+1 \text { i } 1=2 i^{i \pm}\left(f^{0}\right)\right) ; i+1 i{ }^{1=2} i^{i}{ }^{ \pm}\left(f^{0}\right)\right)<i \frac{ \pm}{4}
$$

This implies that $G\left(x_{p}\left(i+{ }^{1} i^{1=2} s\right) ; i+{ }^{1} i 1=2 s\right)$ changes the sign within the interval $\left(i^{ \pm}\left(f^{0}\right) ; i^{i \pm}\left(f^{0}\right)\right)$ : Due to the uniform convergence and the representation (13) there are no other sign inversions at $s<i^{ \pm}\left(f^{0}\right)$ : T herefore, there exists

$$
S^{x}\left({ }^{1}\right) 2\left(i^{ \pm}\left(f^{0}\right) ; i^{i} \pm(f 0)\right)
$$

satisfying the ...rst equality in (12). The inequality for derivative is evident and convergence of $s^{\not \alpha}\left({ }^{1}\right)$ to $s^{\not \alpha}$ follows from (14) and the arbitrariness of \pm

Remark 2: T his theorem shows that the limit system (10) dem onstrates almost the same behavior as the original one for suф ciently large ${ }^{1}$ : T he main result is the following Corollary which establishes the single jump representation with the aid of the limit system solution.

Corollary 1: For suф ciently small " > 0 on the interval $[0 ; i+")$; solution of the original system (2), (3) converges to some discontinuous functions $\left(x_{p}(t) ; x_{v}(t)\right)$, such that

$$
x_{p}(t)=x_{p}(t) ; \quad x_{v}(t)=x_{v}(t) ; \quad t<i ;
$$

and

$$
x_{p}(i+)=\lim _{1 " 1} x_{p}\left(i+1 ; 1=2 S_{1}^{\alpha}\right)=x_{p}(i) ; \quad x_{v}(i+)=\lim _{111} y_{v}\left(i+1 ; 1=2 S_{1}^{\alpha}\right)=y_{v}\left(S^{\alpha}\right):
$$

Remark 3: A ssumption 1 of Theorem 1 can be relaxed as follows, (cf. [50], Theorem 7 §1)

$$
\begin{aligned}
& \lim _{\lim _{1} 1}^{Z s} 11=2 F_{v}^{s} \frac{y_{p} i x_{p}}{11=2}+x_{p} ; y_{v} ; w_{1}(u) ; i+1 ; 1=2 u^{1} d u= \\
& Z_{0}^{s} F_{v}^{s}\left(y_{p} ; y_{v} ; u ; w_{1}(u) ; x_{p} ; i\right) d u ; \\
& 0
\end{aligned}
$$

for any $y_{p} ; y_{v}$ uniformly on $\left[0 ; s^{\ltimes}+"\right)$ : Condition (15) is much weaker than the Lipschitz one, but it is also suф cient for the uniform convergence.

Remark 4: If in the assumption 2 of Theorem $1 "=0$, one can establish only the existence of sequence $s^{\ltimes}(1)$ ! $S^{\not}$ such that

$$
\lim _{1 ! 1} G\left(x_{p}\left(i+1 i 1=2 S^{\not \alpha}(1)\right) ; i^{1} i^{1=2} S^{\not \alpha}(1)\right)=0:
$$

However, the Corollary 1 is still valid. 


\section{B. Repr esent at ion of the Singl e-J ump Gener al ized Sol ut ion}

Let the system start from the initial condition $x_{p}(0) ; x_{v}(0)$ such that $G\left(x_{p}(0) ; 0\right)<0$ and $i$ be the ..rst point where the system engages the constraint, so that conditions (4) and (5) hold. In order to describe the discontinuity at a point $i$, introduce the dixerential equation

$$
\mathrm{y}_{\mathrm{p}}(\mathrm{s})=\mathrm{F}_{\mathrm{p}}\left(\mathrm{y}_{\mathrm{p}}(\mathrm{s}) ; \mathrm{y}_{\mathrm{v}}(\mathrm{s}) ; \mathrm{u}(i) ; \mathrm{x}_{\mathrm{p}}(\dot{i}) ; i\right) ; \quad \mathrm{y}_{\mathrm{v}}(\mathrm{s})=\mathrm{F}_{\mathrm{v}}\left(\mathrm{y}_{\mathrm{p}}(\mathrm{s}) ; \mathrm{y}_{\mathrm{v}}(\mathrm{s}) ; \mathrm{s} ; \mathrm{w}_{i}(\mathrm{~s}) ; \mathrm{x}_{\mathrm{p}}(i) ; i\right) ; \quad \mathrm{s} 2[0 ; 1)
$$

with initial condition $y_{p}(0)=x_{p}(i) ; \quad y_{v}(0)=x_{v}(i)$ and control signal

$$
w_{i}(s) 2 W^{1 / 2} R^{k} \text {. }
$$

Let

$$
\underline{a}\left(\phi w_{i}(\phi ; i)=@_{\underline{a}_{v}\left(\phi w_{i}(\phi ; i)\right.}^{0} A\right.
$$

denote a shift operator along the paths of (16). Then the discontinuity of the path $x_{v}(\Phi$ at instant $t=i$ can be viewed as the result of the action of this shift operator and described by

$$
x_{v}(i)=x_{v}(i i)+\underline{a} v\left(x_{p}(i) ; x_{v}(i i) ; w_{i}(\phi ; i):\right.
$$

Relation (19) describes the jump at $t=i$ in terms of the equation (16) (the equation of "fast dynamics"), so that if

$$
\odot\left(\phi s ; w(\phi ; i)=@ \begin{array}{c}
\Theta_{p}\left(\phi s ; w_{i}(\phi ; i)\right. \\
\Theta_{v}\left(\phi s ; w_{i}(\phi ; i)\right.
\end{array}\right.
$$

is the general solution of (16) with initial condition $y_{p}(0)=x_{p}(i), \quad y_{v}(0)=x_{v}(i i)$, then

$$
x_{v}(i)=\bigodot_{v}\left(x_{p}(i) ; x_{v}(i i) ; s^{\alpha}\left(x_{p}(i) ; x_{v}(i i)\right) ; w_{i}(\phi) ; i\right) ;
$$

where

$$
s^{\mathfrak{x}}\left(x_{p}(i) ; x_{v}(i i)\right)=
$$

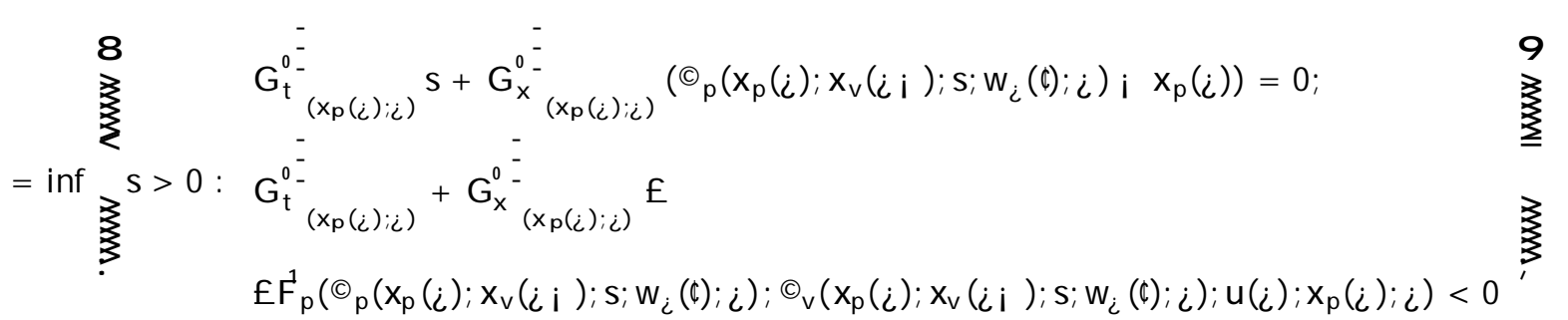

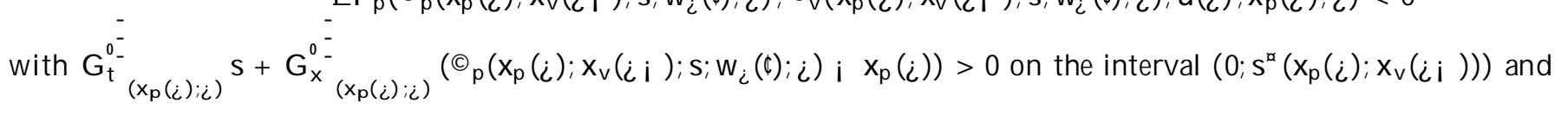

$$
\underline{a}_{v}\left(x_{p}(i) ; x_{v}(i i) ; w_{i}(\phi ; i):=\Theta_{v}\left(x_{p}(i) ; x_{v}(i i) ; s^{\not a}\left(x_{p}(i) ; x_{v}(i i)\right) ; w_{i}(\phi ; i) \text { i } x_{v}(i i):\right.\right.
$$

Remark 5: The shift-operator jump representation (23) is admissible for any unconstrained discr ete-continuous system that has trajectories robust with respect to variation of impulsive input [36], [38], [58]. For systems with constraints, however, one can not guarante at the outset that the shift-operator jump representation exists for any initial conditions $x_{p}(i) ; x_{v}(i i)$ and the impulsive control; $w_{i}(\phi)$, since the existence of $s^{\mathbb{a}}\left(x_{p}(i) ; x_{v}(i i)\right)<1$ can not guaranteed. As follows from the Assumption 2 of Theorem 1 , one needs the constraints to be "repulsive". In the next subsection, a useful su $\emptyset$ cient condition for the existence of the shift-operator jump representation is given; this condition, however, will be assumed to hold from this point on.

De..ne the generalized solution of system (2), (3) as a pointwise limit of ordinary solution if ${ }^{1}$ ! 1 . Then, by using the Corollary 1 and the shift-operator jump representation introduced above, the following is easily der ived:

Theorem 2: The generalized solution $f x_{p}(t) ; x_{v}(t) g$ satis.. es on the interval $[0 ; i+")$ the system of nonlinear generalized dixerential equations

$$
\begin{aligned}
& \underline{x}_{p}(t)=F_{p}^{r}\left(x_{p}(t) ; x_{v}(t) ; u(t) ; t\right) ; \\
& \left.\underline{x}_{v}(t)=F_{v}^{r}\left(x_{p}(t) ; x_{v}(t) ; u(t) ; t\right)+\underline{a}_{v}\left(x_{p}(i) ; x_{v}(i i) ; w_{i}(d) ; i\right) \sharp t \quad i \quad j\right) .
\end{aligned}
$$




\section{The Sufficient Condit ion for the Const raint s to be Repul sive}

The assumption 2 of Theorem 1 means that "force" $F$ has the property to repulse the system from the inhibited domain. Let us express this property in terms of the so-called restitution force. Consider the motion in the area $f\left(\dot{y}_{p} ; s\right): G_{x}^{0}\left(x_{p}(i) ; i\right)\left[y_{p} ; x_{p}(i)\right]+G_{t}^{O}\left(x_{p}(i) ; i\right) s>0 g$ along the paths of the limit system (10). The term

$$
Z\left(y_{p}(s) ; s\right)=G_{x}^{0}\left(x_{p}(i) ; i\right)\left[y_{p}(s) ; \quad x_{p}(i)\right]+G_{t}^{0}\left(x_{p}(i) ; i\right) s
$$

characterizes the principal part of constraint violation, whereas the restitution force is typically expressed in terms of $Z$; as $F(s)=\frac{d^{2}}{d s^{2}} Z(s)$. Suppose that one can ascertain that this restitution force is of the visco-elastic type and such that

$$
\frac{d^{2}}{d s^{2}} Z(s) \quad \text { i } k_{1} Z(s) \text { i } k_{2} Z(s) \text { : }
$$

O ne can then obtain the following criterion, the proof of which is given in the A ppendix.

Proposition 1: Let the restitution force satisfy (25) in the area $Z>0$ : Let also $k_{1} ; k_{2}, 0$ and satisfy the inequality

$$
\mu_{\frac{k_{2}}{2}} \bigcap_{2}<k_{1} \text { : }
$$

Suppose that $Z(0)=0$ and $Z(0)>0:$ Then there exists

$$
s^{\alpha}=\operatorname{inff} s>0: Z\left(s^{\not}\right)=0 ; Z\left(s^{\alpha}\right)<0 g<1:
$$

Remark 6: This proposition means that the restitution force (25) guarantees the repulsion in the ..nite time. Thus, if the limit system (10) satis..es the assumption 2 of Theorem, then the constraint is repulsive.

\section{Model ing of Systems with Multipl e Cont rol led Singularities}

\section{A. System Model with Multipl e J umps}

Having obtained the single jump represent ation given by Theorems 1 and 2 , consider a discrete-continuous dynamical system with behavior representable on the int erval $[0 ; T]$ by a pair of the piecewise continuous functions $\left(x_{p}(t) ; x_{v}(t)\right) 2$ $R^{n} \in R^{n}$, that satis.. es the dixerential equation

$$
\underline{x}_{p}(t)=F_{p}^{r}\left(x_{p}(t) ; x_{v}(t) ; u(t) ; t\right) ; \underline{x}_{v}(t)=F_{v}^{r}\left(x_{p}(t) ; x_{v}(t) ; u(t) ; t\right) ;
$$

with a given initial condition $\left(x_{p}(0) ; x_{v}(0)\right) 2 R^{n} f R^{n}$. In $(27)\left(x_{p}(t) ; x_{v}(t)\right)$ are the generalized state and velocity

$$
\mathrm{u}(\mathrm{t}) 2 \mathrm{U} \frac{1 / 2}{2} \mathrm{~m}^{\mathrm{m}} \text {; }
$$

where $\mathrm{U}$ is some compact set and $\mathrm{u}(\mathrm{t})$ is a control signal. T his equation describes the continuous system evolution until the attainment of the switching surface de..ned by the relation

$$
\mathrm{G}\left(\mathrm{x}_{\mathrm{p}}(\mathrm{t}) ; \mathrm{t}\right)=0:
$$

In (27) and (29) functions $\mathrm{F}_{p}^{r}\left(\mathrm{x}_{\mathrm{p}} ; \mathrm{x}_{v} ; \mathrm{u} ; \mathrm{t}\right) ; \mathrm{F}_{v}^{r}\left(\mathrm{x}_{\mathrm{p}} ; \mathrm{x}_{v} ; \mathrm{u} ; \mathrm{t}\right)$ and $\mathrm{G}\left(\mathrm{x}_{\mathrm{p}} ; \mathrm{t}\right)$ are continuous in all variables, and $\mathrm{F}_{\mathrm{p} ; \mathrm{v}}^{r}$ are Lipschitzian with respect to $\left(x_{p} ; x_{v}\right)$; so that

$$
\mathrm{KF}_{\mathrm{p} ; \mathrm{v}}^{\mathrm{r}}\left(\mathrm{x}_{\mathrm{p}}^{1} ; \mathrm{x}_{\mathrm{v}}^{1} ; \mathrm{u} ; \mathrm{t}\right) \text { i } \mathrm{F}_{\mathrm{p} ; \mathrm{v}}^{\mathrm{r}}\left(\mathrm{x}_{\mathrm{p}}^{2} ; \mathrm{x}_{\mathrm{v}}^{2} ; \mathrm{u} ; \mathrm{t}\right) \mathrm{k} \quad L\left(k \mathrm{x}_{\mathrm{p}}^{1} \mathrm{i} \mathrm{x}_{\mathrm{p}}^{2} \mathrm{k}+\mathrm{k} \mathrm{x}_{\mathrm{v}}^{1} \mathrm{i} \mathrm{x}_{\mathrm{v}}^{2} \mathrm{k}\right)
$$

for any $\left(x_{p}^{1} ; x_{v}^{1}\right) 2 R^{n} £ ; R^{n} ;\left(x_{p}^{2} ; x_{v}^{2}\right) 2 R^{n} £ ; R^{n} ;$ u $2 U$; t $2[0 ; T]$ with some positive constant $L<1$ : Therefore, equation (27) has the unique solution for any L ebesgue measurable control $u(\phi$ de..ned on the whole interval $[0 ; T]$ with an arbitrary initial condition $\mathrm{x}_{0}$.

Suppose that originally $G\left(x_{p}(0) ; 0\right)<0$; and de..ne a sequence of the intersection times

$$
0<i_{1}<:::<i<:::<T
$$

as follows:

$$
i_{1}=\begin{aligned}
& \stackrel{8}{\gtrless} \inf _{0<\mathrm{t} T} \mathrm{ft}: \mathrm{G}\left(\mathrm{x}_{\mathrm{p}}(\mathrm{t}) ; \mathrm{t}\right)>0 \mathrm{~g} ; \\
& ? \quad 1 ; \text { if the set is empty; }
\end{aligned}
$$


and

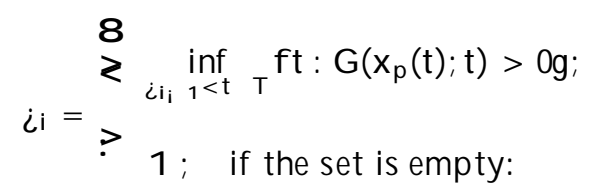

As it is seen, $\dot{\alpha} ; i=1 ; 2 ;::$ are the exit times from the domain $f\left(x_{p} ; t\right): G\left(x_{p} ; t\right) \quad 0 g$, and since the path $x_{p}(\phi$ is

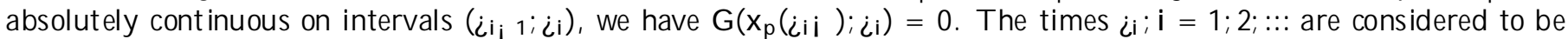
the times of the path singularity, i.e. the path $x_{v}\left(\Phi\right.$ is considered to be discontinuous at the instant $t=i_{i}$.

\section{B. Repr esent at ion of the Gener al ized Sol ut ion wit h Mul t ipl e J umps}

Using the shift-operator single jump representation introduced earlier to describe discontinuity at the instant $t=i_{i}$ yields

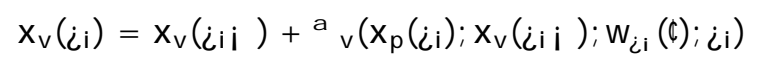

with

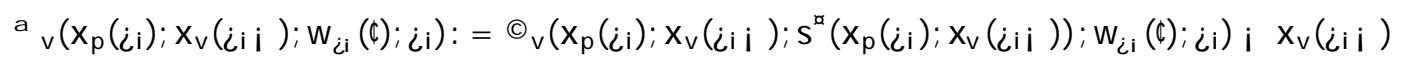

where functions $\underline{a}_{v} ; \bigcirc_{v}$ are de..ned by relations (23), (21), (22). Then, the single-jump description of the discretecontinuous system can be generalized to the case of multiple jumps shown in Figure 5 as follows.

Theorem 3: Suppose that on the interval $[0 ; \mathrm{T}]$ a control signal $u(t) 2 \mathrm{U}$ is given, the set of instances

$$
0<i_{1}<:::<i_{1}<:::<\mathrm{T} ; \mathrm{i} \quad \mathrm{N}<1
$$

is de..ned that satis..es (32), and the appropriate shift-operators ${ }^{a}{ }_{v}\left(x_{p}\left(\dot{\nu}_{i}\right) ; x_{v}\left(\dot{c i}_{i} i\right) ; w_{i j}\left(\phi i i_{i}\right)\right.$ augmented by the impulsive control functions $w_{i \mathrm{i}}(\Phi)$ are well-de..ned for each $\dot{i}$. Then, if 1 ! 1 , the corresponding sequence of ordinary solutions of the system (2), (3) converges everywhere on $[0 ; T]$ (except, possibly, at the points $\left\{\mathrm{i} i g_{i}\right.$ ) to the generalized solution $f x_{p}(t) ; x_{v}(t) g$ that satis..es on the interval $[0 ; T]$ the system of nonlinear generalized dixerential equations

$$
\begin{aligned}
& \underline{x}_{p}(t)=F_{p}^{r}\left(x_{p}(t) ; x_{v}(t) ; u(t) ; t\right) ;
\end{aligned}
$$

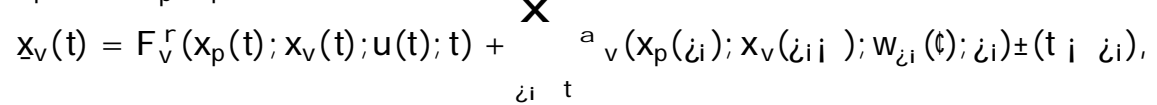

or the corresponding equivalent integral form

$$
\begin{aligned}
& x_{p}(t)=x_{p}(0)+{ }_{0}^{R} F_{p}^{r}\left(x_{p}(s) ; x_{v}(s) ; u(s) ; s\right) d s ;
\end{aligned}
$$

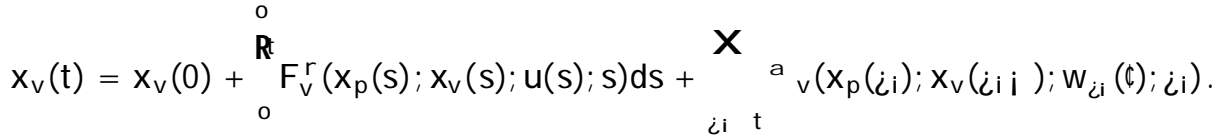

Proof: The proof of this theorem directly follows from Theorem 2 by sequentially taking the limit on each

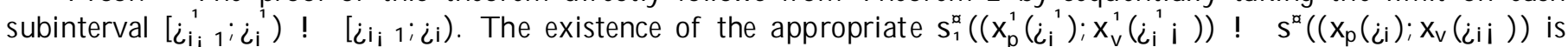
ascertained through transversality of all the paths hitting the constraints to the corresponding constraint surfaces and the standard arguments of the continuous dependence of the solution on the initial conditions. The shift-operator representation of jumps al so implies the Lipschitzian character of function ${ }^{a}{ }_{v}$, thereby guaranteeing the existence and uniqueness of the solution of (35). The details of the proof are standard and are omitted.

Remark 7: In this description, the state of the system changes continuously on half-intervals $\left[0 ; i_{1}\right), \ldots\left[i_{i} i, i i_{i}\right) ; \ldots$ $\left[i_{N} ; T\right.$ ], and undergoes a sudden change at every instant $\Sigma_{i}$. Due to equation (33), the values of these changes depend on the state immediately preceding the jump and the impulsive control signal $w_{i i}(\phi)$ applied during the singularity phase corresponding to the instant $i$.

In the next section it is shown how this machinery can be applied to the analysis of the typical mechanical systems with constraints.

Remark 8: Theorem 3 demonstrates that the solutions of the original system (2), (3) and the system of nonlinear generalized dixerential equations (35) (limit representation) can be made arbitrarily close to each other in a weak-* topology of the space of functions of bound ed variation by the appropriat e choice of the value of the elasticity coet cient 1 . 


\section{Real izat ion of the impul sive cont $r$ ol}

The signi..cance of T heorem 3 is in admitting the realization of the desired generalized solutions and the corresponding impulsive control signals obtained on the basis of the limit system in the corresponding original system. Suppose that the generalized solution $f x_{p}(t) ; x_{v}(t) g$, the corresponding control signal $u(t) 2 U$, the ... nite set of instants $\left\{\dot{z}_{i} g 2[0 ; T)\right.$, and the set of the corresponding control functions $\left\{w_{i j}(s) 2 \mathrm{Wg}\right.$ de..ned for each $\dot{\alpha}$, have all been obtained on the basis of the limit system representation. For a given ..nite ${ }^{1}<1$, by solving system (2) let us de..ne $\dot{i}_{1}^{1}=i_{1}$ and set $f x_{p}^{1}\left(i_{1}^{1}\right) ; x_{v}^{1}\left(i_{1}^{1}\right) g=f x_{p}\left(i_{1}\right) ; x_{v}\left(i_{1}\right) g$ and then solve system (3) for $t, i_{1}$ with controls

$$
\mathrm{u}(\mathrm{t}) ; \mathrm{w}\left(\mathrm{t} ;{ }^{1}\right)=\sum_{0 ;}^{8} \mathrm{w}_{i 1}^{\mu} \frac{\mathrm{t}_{\mathrm{i} i}{ }_{1}}{1 \mathrm{i} 1=2} ; \text { if } \mathrm{t}, i_{1} ; ;
$$

until the instant $i_{1}^{\mathrm{a}}\left({ }^{1}\right)$ satisfying the condition

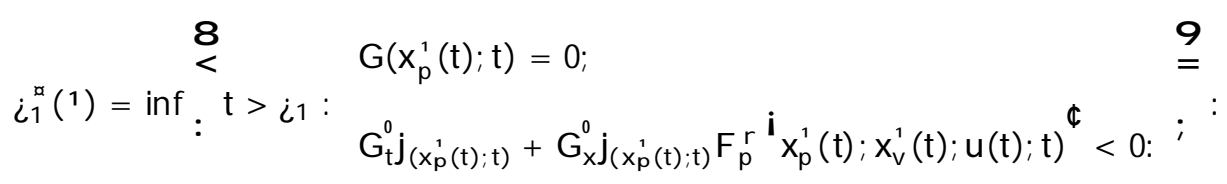

According to Theorem $1 i_{1}^{\alpha}\left({ }^{1}\right) ! \quad i_{1}$ and $f x_{p}^{1}\left(i_{1}^{\alpha}\left({ }^{1}\right)\right) ; x_{v}^{1}\left(i_{1}^{a}\left({ }^{1}\right)\right) g ! f x_{p}\left(i_{1}\right) ; x_{v}\left(i_{1}\right) g$ as ${ }^{1} ! 1$. Then, continue the solution as that of system 2 for $t, i_{1}^{\alpha_{1}}\left({ }^{1}\right)$. A s follows from the continuity of the solutions of dixerential equations, there exists $i_{2}^{1}$ satisfying the condition

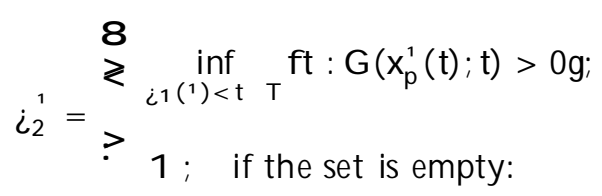

such that $\dot{i}_{2}^{1} ! \quad i_{2}$ and $f x_{p}^{1}\left(\dot{i}_{2}^{1}\right) ; x_{v}^{1}\left(\dot{i}_{2}^{1}\right) g ! f x_{p}\left(i_{2}\right) ; x_{v}\left(i_{2} i\right) g a^{1} ! 1$. Then, repeat the procedure described above with the control signals de.ned for $t, i_{2}$ as follows:

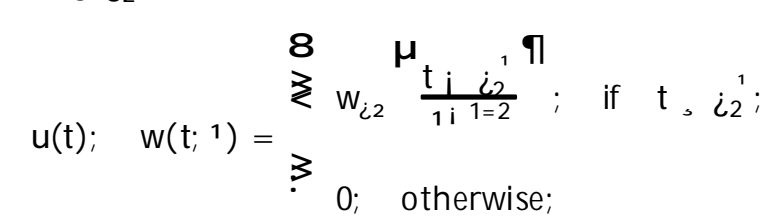

and so on. Then, according to Theorem 3 , the resulting solution $\left\{x_{p}^{1}(t) ; x_{v}^{1}(t) g ! f x_{p}(t) ; x_{v}(t) g\right.$, i.e., the solution of the original system converges to that of the limit system, uniformly in ${ }^{1}$ as ${ }^{1} ! 1$ at all points of continuity.

VI. Mult i-Scal e and Limit Representation of Mechanical Syst ems with Control I ed Const raint s: an anal ysis of mot ivating exampl es

Let us apply the description of motion given by the theorems formulated in the previous section to two mechanical systems introduced in Section II.

\section{A. Example 1: Coll ision Mapping in the Motion of a Ball Coll iding with a Moving Racket}

In Example 1 given Section II, the system has the state vector $z=\left(x_{p}^{1} ; x_{p}^{2} ; x_{p} ; x_{v}^{1} ; x_{v}^{2} ; X_{v}\right)$; where $\left(x_{p}^{1} ; x_{p}^{2}\right)$ are the horizontal and the vertical coordinates of the moving ball, $\left(X_{v}^{1} ; x_{v}^{2}\right)$ are the corresponding velocities, and $\left(X_{p} ; X_{v}\right)$ are the coordinate and velocity of the obstacle surface in the vertical direction. Then, the constraint which de..nes the free motion area is given by the relation

$$
G(z)=X_{p} i \quad x_{p}^{2} \quad 0:
$$

1. General motion equations. First, consider the motion along the vertical axis only. Suppose that in the area free of constraint the motion is described by the equations

$$
\begin{array}{ll}
\underline{x}_{p}^{1}(t)=x_{v}^{1}(t) ; \quad \underline{x}_{v}^{1}(t)=0 ; \\
\underline{x}_{p}^{2}(t)=x_{v}^{2}(t) ; \quad \underline{x}_{v}^{2}(t)=i g ; \\
x_{p}(t)=x_{v}(t) ; \quad x_{v}(t)=\frac{F(t)}{M}:
\end{array}
$$


In the inhibited area the motion is described by the equation

$$
\begin{aligned}
& \underline{x}_{p}^{1}(t)=x_{v}^{1}(t) ; \quad \underline{x}_{v}^{1}(t)=i \text { o1 } \frac{F^{s}\left(z(t) ;^{1}\right)}{m} \text { sign } x_{v}^{1}(s) ; \\
& \underline{x}_{p}^{2}(t)=x_{v}^{2}(t) ; \quad \underline{x}_{v}^{2}(t)=i g_{i}{ }^{1} \frac{F^{s}\left(z(t) ;{ }^{1}\right)}{m} ; \\
& X_{p}(t)=X_{v}(t) ; \quad X_{v}(t)=\frac{F(t)}{M}+1 \frac{F^{s}\left(z(t) i^{1}\right)}{M}:
\end{aligned}
$$

where

${ }^{2} \mathrm{~F}^{\mathrm{S}}\left(\mathrm{z}(\mathrm{t}) ;{ }^{1}\right)$ is a visco-elastic force during the contact of ball and racket described by the relation

$$
F^{s}\left(z(t) i^{1}\right)=i\left(x_{p}^{2}(t) \text { i } X_{p}(t)\right) \text { i } 2^{1} \text { i } 1=2 \gg\left(x_{v}^{2}(t) \text { i } X_{v}(t)\right) ;
$$

$2 \mathrm{~m} ; \mathrm{M}$ are the masses of ball and racket, respectively,

$2 \mathrm{~g}$ is the gravitational acceleration,

2 o 0 is the dry friction coec cient,

$2 \mathrm{~F}(\mathrm{t})$ such that $\mathrm{jF}(\mathrm{t}) \mathrm{j} \quad \mathrm{F}_{0}<1$, is an external control force acting on the racket,

$21>0$ is the elasticity coec cient and $0 \gg 1$ is the damping.

Equations (42),(43) describe the continuous motion in case of ${ }^{1}<1$. The objective, however, is to obtain the velocity jump representation corresponding the limit motion as ${ }^{1}$ ! 1 :

2. J ump representation in the vertical direction. Since the motion in the vertical direction does not depend on coordinates $\left(\mathrm{x}_{\mathrm{p}}^{1} ; \mathrm{x}_{\mathrm{v}}^{1}\right)$ one can obtain the jump representation independently. $\mathrm{F}$ irst, consider the motion in the vertical direction with the corresponding reduced state vector $z(t)=\left(x_{p}^{2} ; X_{p} ; X_{v}^{2} ; X_{v}\right)$. A pplying Theor em 1 and calculat ing $F$ by formula (9) yields the following system (cf. eq. (10)) for new variables ( $\dot{y}_{p}^{2}(s) ; \psi_{p}(s) ; \psi_{v}^{2}(s) ; \psi_{v}(s)$ ) describing the motion in the enlarged space-time scale:

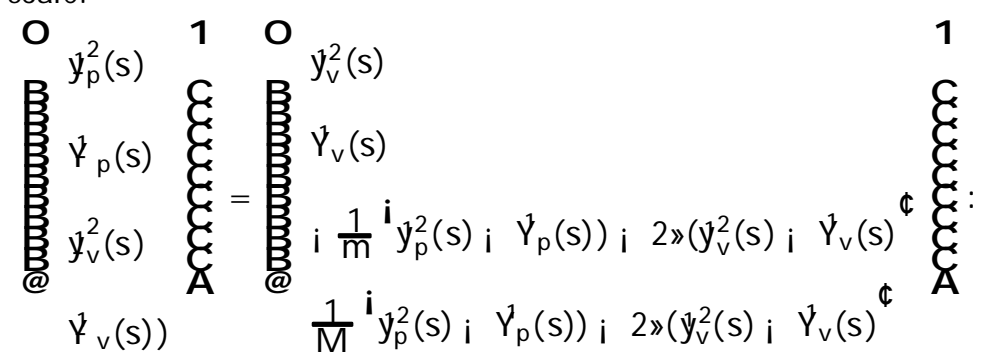

The system (45) has to be solved for $s>0$ with initial conditions

$$
y_{p}^{2}(0)=\Psi_{p}(0)=x_{p}^{2}(\dot{c}) ; \quad y_{v}^{2}(0)=x_{v}^{2}(\Sigma)>\Psi_{v}(0)=X_{v}(i) ;
$$

until the exit time de..ned by the relation (11)

$$
\mathrm{s}^{\alpha}=\text { inf }{ }_{:}^{8} \mathrm{~s}>0: \begin{aligned}
& \mathrm{\psi}_{\mathrm{p}}^{2}(\mathrm{~s}) \text { i } \mathrm{\psi}_{\mathrm{p}}(\mathrm{s})=0 ; \stackrel{9}{=} \\
& \mathrm{\psi}_{\mathrm{v}}^{2}(\mathrm{~s}) \text { i } \mathrm{\psi}_{\mathrm{v}}(\mathrm{s})<0
\end{aligned} ;:
$$

The variable

$$
Z(s)=\psi_{p}^{2}(s) \text { i } \vec{Y}_{p}(s) ;
$$

which characterizes the constraint violation satis.. es the equation

$$
\frac{d^{2}}{d s^{2}} Z(s)=\frac{M+m}{M m} \text { i } Z(s) \text {; } 2 \gg \frac{d}{d s} Z(s) \text {; }
$$

with initial conditions

$$
Z(0)=0 ; \quad \frac{d}{d s} Z(0)>0:
$$

Therefore, according to Proposition 1 this constraint is repulsive if the condition

$$
j \gg j<\frac{m M}{M+m}^{\bigcap_{1=2}}=\frac{1}{k}
$$


holds. In this case there exists

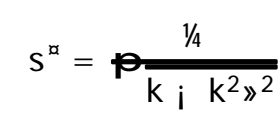

satisfying (47) and by Corollary 1 one can calculate the jumps of variables $X_{v}^{2}(i)$ and $X_{v}(i)$ : They are equal to

$$
\begin{aligned}
& \pitchfork x_{v}^{2}(i)=i \frac{M\left(1+k_{r}\right)}{M+m}{ }^{f} x_{v}^{2}(i i) i X_{v}(i i)^{\not a} ; \\
& \phi X_{v}(i)=\frac{m\left(1+k_{r}\right)}{M+m}{ }^{f} x_{v}^{2}(i i) i X_{v}(i i)^{\not a} ;
\end{aligned}
$$

where the restitution coec cient $k_{r}$ is equal to

$$
k_{r}=\exp i \frac{1 / k »}{\frac{k i k^{2} »^{2}}{2}} \text { : }
$$

3. Motion in the horizontal di rection with dry friction. B y using the space-time transformation of $\left(x_{p}^{1} ; x_{v}^{1}\right)$ we obtain the following system of equations in new space-time scale

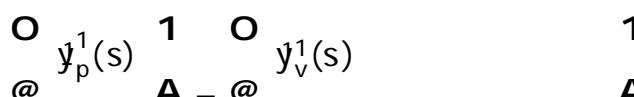

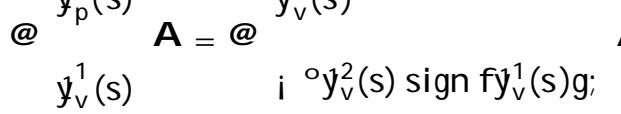

where by de..nition sign $\mathrm{fg}=0$. Solution of $(50)$ has the form

$$
\begin{aligned}
& \min \not \mathbf{Z} ; i^{x} g
\end{aligned}
$$

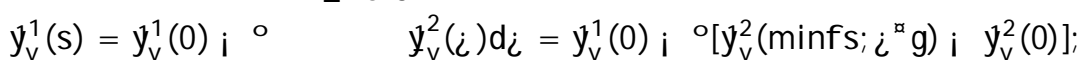

$$
\begin{aligned}
& 0
\end{aligned}
$$

where

$$
i^{\infty}=\operatorname{inff}_{i}>0: y_{v}^{1}(\dot{i})=0 \mathrm{~g}
$$

T herefore,

$$
\psi_{v}^{1}\left(s^{\alpha}\right)=y_{v}^{1}(0) \text { i o }\left[y_{v}^{2}\left(\operatorname{minf} s^{\alpha} ; i^{\alpha} g\right) \text { i } y_{v}^{2}(0)\right] ;
$$

and taking into account the initial condition $\mathrm{b}_{v}^{1}(0)=\mathrm{x}_{v}^{1}(\mathrm{ii})$ by Corollary 1 one can calculate velocity increment in the horizontal direction as

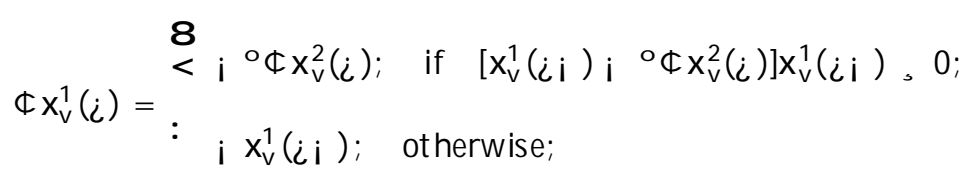

where $\phi x_{v}^{2}(\dot{c})$ is de..ned by the relation (49).

The next example shows another type of introduction of the impulsive action int o the singular phase.

\section{B. Exampl e 2: I mpact with the rot at ing surface}

Let the dynamics of the ball be described by a pair of vectors $\left(x_{p} ; x_{v}\right)$; where $x_{p} 2 R^{3}$ correspond to the position and $\mathrm{x}_{v} 2 \mathrm{R}^{3}$ to the velocity, so that the nonsingular phase is described by the equation

$$
\underline{x}_{p}(t)=x_{v}(t) ; \quad \underline{x}_{v}(t)=0:
$$

The position of the racket surface plane is given by the relation $\left\langle\mathrm{y} ; \mathrm{n}\left(\mathrm{t} ;{ }^{1}\right)\right\rangle=0$; where $\langle\phi, \phi$ is the scalar product, 1 is the elasticity coec cient, and $n(t)$ is a unit vector of normal to the surface. For simplicity, consider the case when the center of the rotation coincides with the point of contact and the rotation axis is orthogonal to the plane formed by vectors $x_{v}$ and $n$. In the singular phase, which takes place whenever the ball hits the surface, the contact force is proportional to the value of the surface deformation and directed perpendicular to the surface, so that if

$$
\mathrm{G}\left(\mathrm{x}_{\mathrm{p}}(\mathrm{t}) ; \mathrm{t}\right)=<\mathrm{x}_{\mathrm{p}}(\mathrm{t}) ; \mathrm{n}\left(\mathrm{t} ;{ }^{1}\right)><0 ;
$$


the equations have the form

$$
\underline{x}_{p}(t)=x_{v}(t) ; \quad \underline{x}_{v}(t)=\mathrm{i}^{1} \mathrm{n}\left(\mathrm{t} ;{ }^{1}\right)<\mathrm{x}_{\mathrm{p}}(\mathrm{t}) ; \mathrm{n}\left(\mathrm{t} ;{ }^{1}\right)>\text { : }
$$

O ne can complete this system with the dixerential equation for $\mathrm{A}\left(\mathrm{t} ;{ }^{1}\right)$

$$
\underline{\mathrm{n}}\left(\mathrm{t} ;{ }^{1}\right)=\mathrm{n}\left(\mathrm{t} ;{ }^{1}\right) f !\left(\mathrm{t} ;{ }^{1}\right)
$$

where a $\mathrm{f}$ b denotes the vector product, and $!\left(\mathrm{t} ;{ }^{1}\right)$ is the angular velocity of the surface rotation. T his velocity could be interpreted as an impulsive control that abruptly changes the angle of the surface during the contact phase. Therefore, this velocity admits the representation

$$
!\left(t ;{ }^{1}\right)=!_{0}^{11=2} w^{\mu} \frac{t_{i} i^{\text {ी }}}{1 \text { i } 1=2}
$$

where $!_{0}$ is the unit vector directed along the rotation axis, $w(\phi$ is an impulsive control, and $i$ is the impact time. The use of the transformation (6)

$$
\begin{aligned}
& y_{p}^{1}(s)=x_{p}(i)+{ }^{1}{ }^{1=2}\left[x_{p}(i+1 i 1=2 s) i x_{p}(i)\right] ; \\
& y_{v}^{1}(s)=x_{v}(i+1 i 1=2 s) ; \quad n^{1}(s)=n\left(i+1 i 1=2 s ;{ }^{1}\right) \\
& t=i+1 i^{1=2} s ;
\end{aligned}
$$

yields the following limit system for limit variables $\left(y_{p}(s) ; y_{v}(s) ; h(s)\right)$ de..ned for $s>0$ :

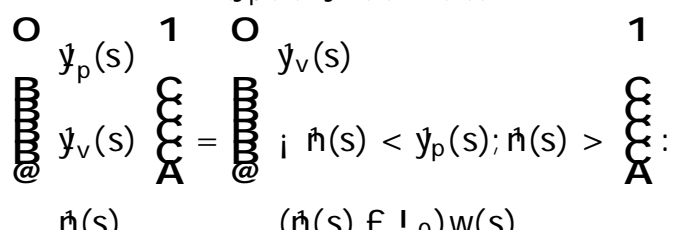

$$
\begin{aligned}
& (h(s) \pm ! 0) w(S)
\end{aligned}
$$

This system has to be solved with the initial conditions

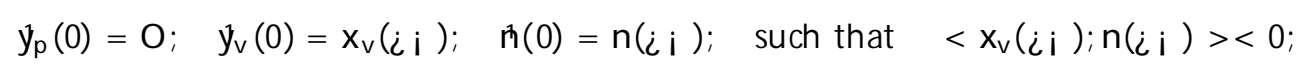

until the time

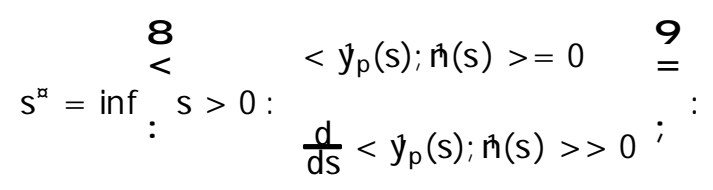

If

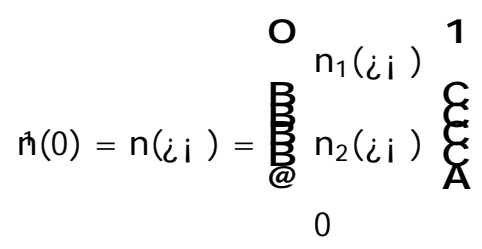

is given, then the solution of the third equation is equal to

$$
\begin{aligned}
& \left.h(s)=\frac{\beta}{\beta} i n_{1}(i i) \sin f A ́(s) g+n_{2}(i i) \operatorname{cosf} A ́(s) g\right\} \\
& 0
\end{aligned}
$$

where

$$
A(s)={ }_{0}^{Z s} w(u) d u
$$




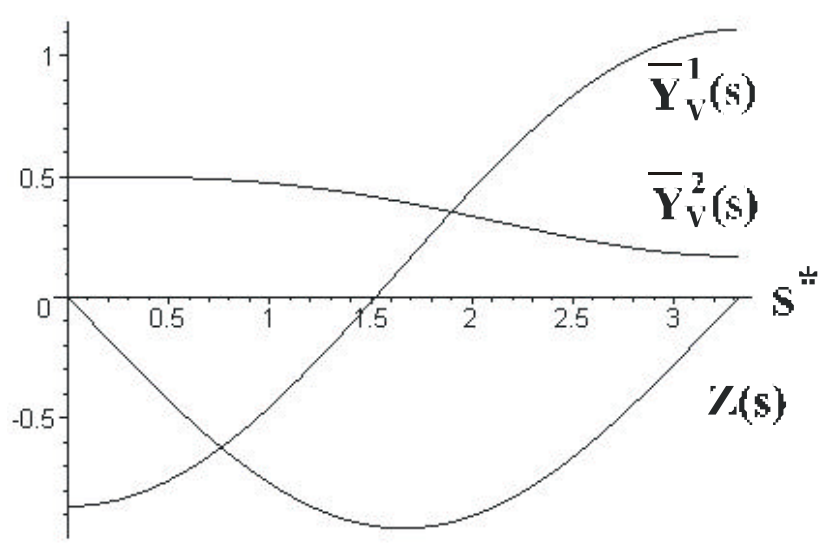

Fig. 6. Solution of the limit system. Clockwise rotation.

is the rotation angle of the contacting surface. Therefore, if the control law $w(\Phi$ is given, one obtains a linear system of dixerential equations

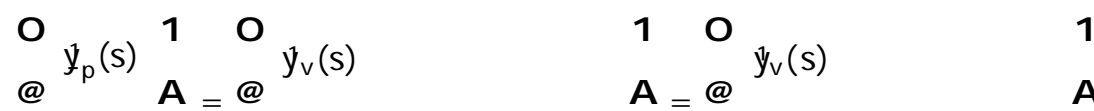

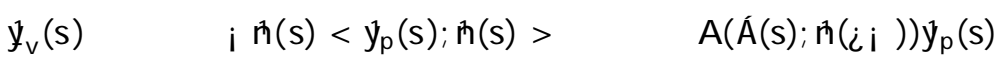

for $\left(\mathbb{y}_{p} ; \mathbb{H}_{v}\right)$; where $A(\dot{A} ; n)$ is some matrix-valued function depending on the current value of the rotation angle, $\dot{A}(s)$, and the initial orientation, $\mathrm{n}(\mathrm{ii})$, of the plane. The general solution of this system has the form

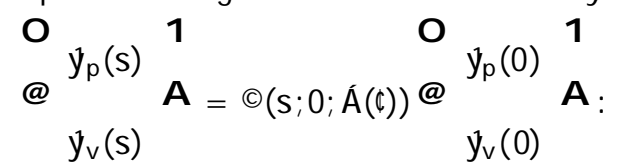

M atrix $\Theta(s ; 0 ; \dot{A}(\Phi)$ can be represented as

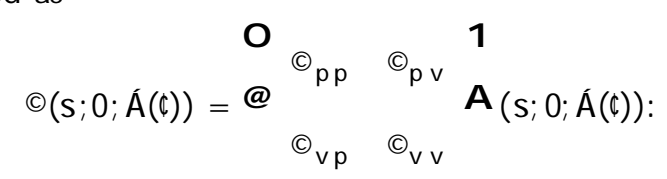

If there exists an exit time $\mathrm{s}^{\sharp}$ de..ned by the relation (54), the collision mapping in this model is then calculated as

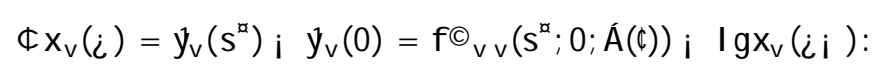

The numerical examples demonstrate the calculation of collision mapping for the following data:

$$
\begin{gathered}
\mathrm{x}_{\mathrm{v}}^{1}(i i)=\mathrm{i} \frac{\mathrm{p} \overline{3}}{2} ; \quad \mathrm{x}_{\mathrm{v}}^{2}(i i)=\frac{1}{2} ; \\
\mathrm{n}^{1}(i)=1 ; \quad \mathrm{n}^{2}(i)=0 ; \\
\mathrm{w}(\mathrm{s})=\mathrm{w}=\mathrm{i} 0: 1 ; \quad \text { and } \quad \mathrm{w}(\mathrm{s})=\mathrm{w}=0: 1:
\end{gathered}
$$

Figures 6 and 7 show the results of the solution calculation of the limit system (55) for positive and negative direction of the surface rotation, respectively.

Using the curves obtained, one can de..ne $s^{\alpha}$ as the ..rst point where $Z(s)=<y(s) ; n(s)>=0$, and then de..ne

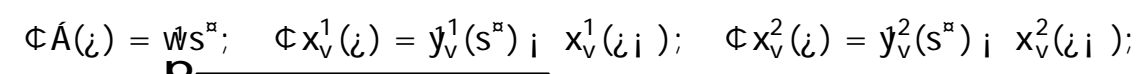

$$
\begin{aligned}
& \phi V(i)=p \frac{p\left(x_{v}^{1}\right)^{1}(i)+\left(\phi x_{v}^{2}\right)^{2}(i):}{}
\end{aligned}
$$




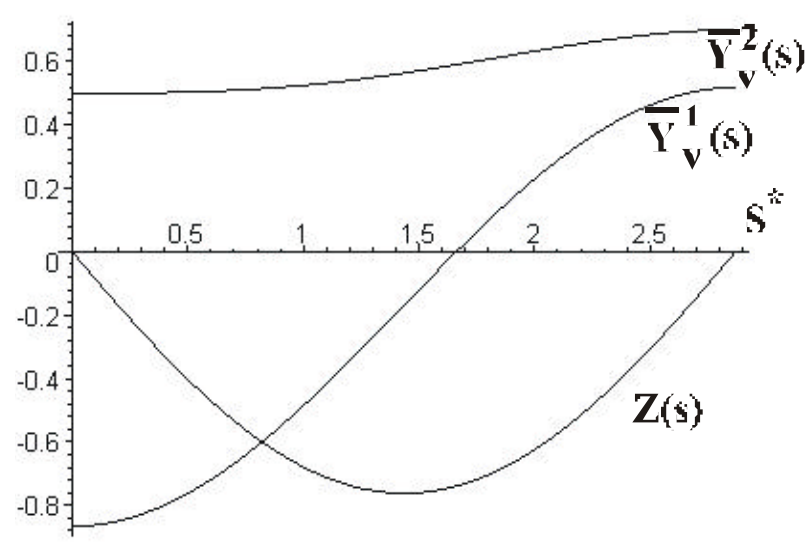

Fig. 7. Solution of the limit system. Anti-clockwise rotation.

In the ..rst case corresponding to the clockwise rotation

$$
s^{\not}=3: 32 ; \quad \pitchfork A ́(i)=\text { i } 0: 332 \mathrm{rad} ; \quad \$ x_{v}^{1}(i)=1: 97 ; \quad x_{v}^{2}(i)=\text { i } 0: 3 ; \$ V(i)=0: 1>0 ;
$$

and in the second case cor responding to the anti-clockwise rotation

$$
s^{\not}=2: 87 ; \quad \pitchfork \dot{A}(i)=0: 287 \mathrm{rad} ; \quad \pitchfork x_{v}^{1}(i)=1: 37 ; \quad x_{v}^{2}(i)=0: 2 ; \pitchfork \vee(i)=\text { i } 0: 12<0:
$$

Thus, fully inclusive as well as simpli..ed representations of systems with controlled collisions are obtained.

\section{Conclusions}

A new class of systems: dynamical systems with controlled singularities is proposed. A subset of this class - systems with controlled elastic impacts - is considered. For the latter subset, a mathematical framework is developed that provides consistent description of controlled impact dynamics and yields control-oriented models suitable for system redesign, motion planning, and control law synthesis. The mathematical framework includes: a system of dixerential equations with unbounded impulsive rhs, or original system, that accommodates controlled collisions, a topological map that regularizes the original system by mapping it into the limit representation, and the limit representation that has bounded control signals and coel cients of the delta-functions in its rhs. It is demonstrated that the corresponding solutions of the original and the limit representations can be made arbitrarily close to each other uniformly except, possibly, in the vicinities of the jump points by the appropriate setting of the value of the elasticity coed cient in the original system. A new type of description of the impulsive action in the form of the cont rolled shift operator along the trajectories of the equation of fast dynamics in the singular phase is introduced and incorporated into the system limit representation; this operator can be viewed as active collision mapping, replacing the traditional one. Implementation of the control laws is shown to be accomplished by simply substituting the time-rescaled bounded control signals found through the use of the limit representation into the actual physical system, resulting in the behavior of the latter close to that of the limit system for suф ciently large values of the elasticity coeф cient and suф ciently accurate original system description.

T wo detailed examples are given which demonstrate the use of the framework developed for the introduction of control actions into singular phase and rigorous derivation of the limit description of the system. The concepts also oxer a much more general and convenient framework for representing various types of mechanical collisions even in the absence of the deliberately introduced impulsive actions.

\section{A ppendix}

Proof: [P roof of Lemma 1]Due to the negativity of derivative at $i^{0}\left(f^{0}\right)$ there exists $\pm 0>0$ such that $i^{\#}\left(f^{0}\right)<$ $i^{0}\left(f^{0}\right)<i^{i}\left(^{\left(f^{0}\right)}\right.$ for any $0< \pm< \pm$ : Moreover, $i^{ \pm}\left(f^{0}\right)$ " $i^{0}\left(f^{0}\right)$; and $i^{i \pm}\left(f^{0}\right) \# i^{0}\left(f^{0}\right)$ if \pm ! 0: Due to the uniform convergence of $f^{n}\left(\phi g\right.$ to $f^{0}(\phi$ for any $\pm< \pm$ one can choose $n( \pm$ such that for $n, n( \pm)$

$$
f^{n}(s)>f^{0}\left(i^{ \pm}\left(f^{0}\right) ; \quad \frac{ \pm}{2}=\frac{ \pm}{2} ; \text { for } s \quad i^{ \pm}\left(f^{0}\right)\right.
$$

and

$$
f^{n}(s)<f^{0}\left(i^{i \pm}\left(f^{0}\right) i \frac{ \pm}{2}+\frac{ \pm}{2} ; \quad \text { for } \quad s=i^{i \pm}\left(f^{0}\right)\right.
$$


Hence by continuity of $f^{n}$ for $n, n(\sharp$; we have

$$
i^{0}\left(f^{n}\right) 2\left(i^{ \pm} ; i^{i \pm}\right)
$$

which establishes the convergence due to the arbitrariness of \pm

P roof: [P roof of $P$ roposition of 1$]$ F unction $Z(s)$ satis..es the equation

$$
\frac{d^{2}}{d s^{2}} Z(s)=f(s) ; k_{1} Z(s) \text { i } k_{2} Z(s) \text {; }
$$

with some function $f(s) \quad 0$ : This equation has an explicit solution

$$
Z(s)=e^{i \cdot s} 4 Z(0) \frac{\sin ! s}{!}+e_{0}^{Z s} e^{i \frac{\sin !(s i i)}{!} f(i) d_{i} 5}
$$

where

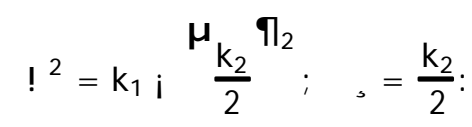

It is easily seen that $z^{i} \underline{1}^{d} \quad 0$ : Consider ..rst the case when the Lebesgue measure

$$
\text { mesfs: } f(s)<0 g>0 \text { : }
$$

Then

$$
\int_{0}^{i z=!} e^{i \sin !\left(s_{i} i\right)} \frac{1}{!} f(i) d i<0
$$

due to positivity of $\sin !(s ; i)$ and negativity of $f$ : Since $Z(0)>0$; there exist an interval, $[0 ; ")$ where $Z(s)>0$ : Therefore, there exists $s^{\natural} 20 ; ?^{4}$ such that $Z\left(s^{\natural}\right)=0$ and $Z(s)>0$ for $s 2\left[0 ; s^{\natural}\right)$ : At the same time

$$
Z\left(s^{\alpha}\right)=e^{i \cdot s^{x}} 4 Z(0) \cos ! s^{\alpha}+e^{z^{x}} e^{i} \cos !\left(s^{\alpha} i \quad i\right) f(i) d_{i} 5 \text {; }
$$

thus taking into account (56) and (57) we obtain

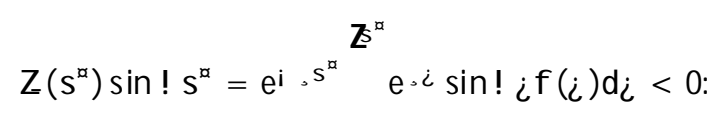

0

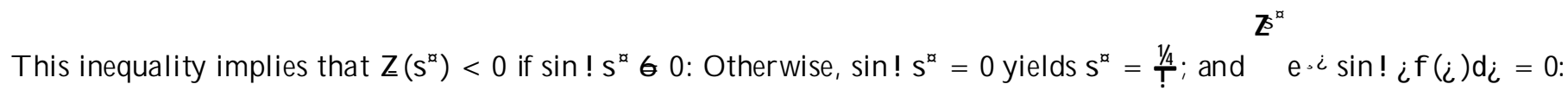
In this case $\mathrm{f}(\mathrm{s})=0$ almost everywhere on $\left[0 ; \mathrm{s}^{\mathrm{x}}\right]$ and as follows from (57)

$$
Z\left(s^{x}\right)=i e^{i \cdot s^{x}} Z(0)<0:
$$

\section{Ref er ences}

[1] V. I. Babitsky, T heory of Vibro-Impact Systems and Applications. Berlin, Germany: Springer-Verlag, 1998.

[2] J. Bentsman and B. M. Miller, "C ontrol of distributed parameter systems with generalized inputs," Autom. and Remote $C$ ontr., vol. 58, no. 7, pp. 65-80, 1997.

[3] J. Bentsman and B. M. Miller, "Control of dynamic systems with unilateral constraints and dixerential equations with measures," Preprints of the 4th IFAC Nonlinear Control Systems Design Symposium 1998 (NOLCOS'98). University of Twente, Enschede, The Netherlands, J uly 1-3, 1998. Eds. H. J . C. Huij berts, H. Nijmeijer, A. J. van der Schaft and J. M. A. Scherpen. Univer sity of Twente, vol. 2, 411-416, 1998.

[4] J. B entsman and B. M. M iller, "Dynamical Systems with Controllable Singularities: Multi-Scale and Limit Representation and Optimal Control," Proc. of the 40th I EE E Conference on Decision and Control 2001 (CD C'01), pp. 3681-3686. O rlan do, Florida, U SA, D ecember, 2001. 
[5] J. Bentsman and B. M. Miller, "M echanical Systems with Unilateral Constraints: Controlled Singul arities A pproach," P roc. of the 40th IEEE Confer ence on Decision and Con trol 2001 (CDC'01), pp. 3692-3697. O rlando, Florida, U SA, D ecember, 2001.

[6] K-F. Bohringer, K. Goldberg, M. Cohn, R. Howe, and A. P isano, "Parallel microassembly with electrostatic force ..elds," Proc. 1998 IEEE International C onference on Robotics and Automation, vol. 2, pp. 1204-1211, 1998.

[7] K-F. B ohringer, B. R. Donald, and N. C. M acD onald, "W hat programmable vector ..elds can (and cannot) do: force ..eld algorithms for ME MS and vibratory plate parts feeders," Proc. 1996 IEE E International C on ference on Robotics and Automation, vol. 1, pp. 822-829, 1996.

[8] K-F. Bohringer, B. R. Donald, R. Mihailovich, and N. C. MacD onald, "A Theory of manipulation and control for microfabricated actuator arrays," Proc. IEEE Micro Electro Mechanical Systems, pp. 102-107, 1994.

[9] A. B ressan an d F. Rampazzo, "On dixerential systems with quadratic impulses and their ap plications to Lagrangian mechanics," SIA M J. Control Optim., vol. 31, pp. 1206-1220, 1993.

[10] A. B ressan, "On the application of control theory to certain problems of Lagrangian systems, and hyper-impulsive motion for these, I,II," Atti. Acc. Lincei Rend. ..S, (8), LXXXII, pp. 91-105, 107-118, 1988.

[11] A. B ressan, "Hyper-impulsive motion and controllable coordinates for Lagrangian systems," Atti. Acc. L incei end. ..s, (8), XIX, pp. $197-246,1989$.

[12] A. B ressan and M. Motta, "A class of mechanical systems with some coordinates as controls. A reduction of certain optimization problems for them. Solution methods," Atti. Acc. Lincei Rend., (9), II, pp. 7-30, 1993.

[13] B. B rogliat o, S. I. N iculescu, and P. O rhant, "On the control of ..nite-dimensional mechanical systems with unilateral constraint," IE EE Trans. Automat. Contr., vol. 42, no. 2, pp. 200-215, 1997.

[14] B. Brogliato and A. Zavala Rio, "On the control of complementary-slackness juggling mechanical systems," IEEE Trans. Automat. Contr., vol. 45, no. 2, pp. 235-246, 2000.

[15] B. B rogliato, Nonsmooth Impact M echanics. Models, Dynamics and Control. L ond on: Springer Communications and Control E ngi neering Series, 2000

[16] F. H. Clarke, Optimi zation and Nonsmooth Analysis. New York: Jonh W iley \& Sons, 1983.

[17] F. H. Clarke and R. B. Vinter, "Optimal multiprocesses," SIA M J . Contr. and O ptim., vol. 27, no. 5, pp. 1072-1091,1989.

[18] M. B. Chiarolla and U. G. Haussmann, "Optimal control of inłation: Central bank problem," SiA M J. Control and Optimiz., vol. 36, no. 3, pp. 1099-1132, 1998.

[19] E. A. Coddington and N. Levinson, Theory of ordinary dixerential equations, New Y ork: McG raw-Hill, 1955.

[20] R. Gabasov, F. M . K irillova, N. V. Balashevich, and J . B ent sman, "Stabilization with the help of bound ed impulse feedbacks," P reprints of the 2nd International Federation of A utomatic Control Symposium on R obust C ontrol D esign, Budapest, H ungary, pp. 143-148, J une 25-27, 1997.

[21] V. A. Dykhta, "Impul sive optimal control in models of economic and quantum electronics." Autom. Remote Contr., vol. vol.60, no. 11, pp.100-112, 1999.

[22] J. W. Grizzle, G. A bba, and F. Plestan, "A symptotically stable walking for biped robots: analysis via systems with impulse exects," IEEE Trans. Automat. Control, vol. 46, no. 1, pp. 51-64, 2000.

[23] A. D. Ioxe and V. M. Tikhomirov, Theory of Extremal Problems. A msterdam: N orth-H olland, 1979.

[24] U. Haussmann and W. Suo, "Singular optimal stochastic control I, II," SIAM J. Contr. and Optim., vol.33, no 3, pp. 916-936 and 937-959, 1995.

[25] R. L. Hipwell, R. S. Muller, and A. P. Pisano, "Characterization of thin-..Jm impact microactuators," Proc. Symp. on Micro-Mech. Syst., 16-21 November, pp. 87-91, 1997.

[26] M. J ean and J . J. M oreau, D ynamics of E lastic or Rigid B odies with Frictional Contact: N umerical M ethods. Publications of Laboratory of M echanics and A coustics, Marseille, A pril, No 124, 1991

[27] P. K undur, Power System Stability and C ontrol. New York: M cG raw-Hill, Inc. 1994.

[28] A. N. K olmogorov and S.V. Fomin, Elements of Theory of F unction and Functional Analysis [in Russian]. M oscow: Nauka, 1976.

[29] V. F. K rotov, V. Z. Bukreev, and V. I. Gurman, N ew M ethods of the Calculus of Variations in Flight Dynamics [in Russian]. Moscow: Mashinostroenie, 1969.

[30] D. Lawden, O ptimal Traj ectories for Space navigation. London: Butterworth, 1963

[31] A. P. Lee, A. P. Pisano, and M. G. Lim, "Impact, friction, and wear testing of microsampl es of polycrystalli ne silicon," Smart M aterials Fabrication and Materials for Micro-E lectro-M echanical Systems, pp. 67-78, 1992.

[32] E. B. Lee and L. Markus, Foundation s of O ptimal Control Theory. New Y ork: J ohn W iley \& Sons, 1967.

[33] N. H. M cClamroch, "Singular systems of dixerential equations as dynamic model for constrained rob ot systems," P roc. of IEEE Int. Conf. on Robotics and Automation, San Francisco, A pr. 8-10, pp. 21-28, 1986

[34] N. H. McClamroch, "A singular perturbation approach to modeling and control of manipulators constrained by a stix environment," Proc. 1989 IEE E Conference on Decision and Control, vol. 3, pp. 2407-2411, Tampa, F L, 1989.

[35] B. M. M iller, "M ethod of discontinuous time change in problems of control of impulse and discrete-continuous systems," Autom. Remote Contr., vol. 54, no. 12, pp. 1727-1750, 1993.

[36] B. M. Miller, "The generalized solutions of nonlinear optimization problems with impulse control," SI AM J. Contr. O ptimiz. vol. 34 no. 4, pp. 1420-1440, 1996.

[37] B. M. M iller, "T he generalized solutions of ordi nary di xerential equation sin the impulse control problems," J . Math. Syst., Est., C ontr., vol. 6, no. 4, pp. 415-435, 1996.

[38] B. M . M iller, "Rep resent ation of robu st and non-robust solutions of nonlinear discrete-cont inuous systems," Proc. International Workshop on H ybrid and Real-T ime Systems (HART' 97), G renoble, France, M arch 26-28, 1997. Ed. O. Maler. Lecture Notes in Computer Sci ence, pp. 228-239, 1201, Springer-V erlag, 1997.

[39] B. M. Miller, "Optimization of generalized sol utions of nonlinear hybrid (discrete-continu ous) systems," P roc. F irst International W orkshop. Hybrid Systems: Computation and Control (HSCC'98), B erkeley, California, USA, A pril 13-15, 1998. Eds. T. A. Henzinger and S. Sastry. Lecture Notes in Computer Science, 1386, Springer-Verlag, pp. 334-345, 1998.

[40] B. M. Miller and J. Bentsman, "A new approach to control of dynamic systems with unilateral constraints," Proc. 14th IFAC W orld Congress, B eijing, China, v. C, pp. 199-203, 1999.

[41] B.M.M iller and J. B entsman, "G eneralized solutions in dynamic systems with unilateral constraints," in: Proccedings of NOLCOS'01, 5th IFAC Symposium "N onlinear Control Systems" Saint-Pet ersbourg, Russia, pp. 625-628, 2001

[42] J. K. M ills and C. Nguyen, "Robotic manipulat or collision: modeling and simulation," Trans. A SME J. Dyn. Sys. M eas. and Contr., vol. 114, no. 4, pp. 650-659, 1993.

[43] E. F. M ishenko and N. H. R ozov, Dixerential Equations with Small Parameter and the Relaxational Oscilations [in Russian]. Moscow: Nauka, 1975.

[44] M. D. P. Monteiro-Marques, Dixerential Inclusions in Nonsmooth Mechanical Problems: Shocks and Dry Friction. Boston, MA: Birkhauser, 1993

[45] J. J. Moreau, "Bounded variation in time," in: Topics in N onsmooth M echanics, Eds: J J. Moreau, P.D.P anagiotopolous, and G.Strang. Basel: B irkhau ser Verlag, pp. 1-74, 1988 
[46] J. J. Moreau, "Unilateral contacts and dry friction in ..nite freedom dynamics", in Nonsmooth Mechanics and Applications, CIM S Course and Lectures, no. 302, W ien: Springer-Verlag, pp. 1-82, 1988.

[47] I. G. N atanson, Theory of Functions of a Real Variable. Vols. 1 and 2, N ew York: Frederick Ungar, 1955, 1960.

[48] Yu. V. Orlov, Theory of Optimal Systems with Generalized Controls. [in Russian], N auka, M oscow (1988).

[49] A. I. Panasyuk and J. B entsman, "A pplication of quasidixerential equations to the description of discontinuous processes," Dixerential Equations, vol. 33, no. 10, pp. $1339-1348,1997$.

[50] A. P h. P hillipov, Dixerential Equations with D iscontinuous Right-Hand Side. D ortrecht, The Netherlands: K luwer, 1988.

[51] F. P feifer and C. Glocker, M ulti-B ody Dynamics with Unilateral Constraints. N ew-York: W iley, 1996.

[52] W. Rudin, Real and Complex Analysis, New York: McGraw-Hill, 1966.

[53] A. Tornambe, "M odeling and control of impact mechanical systems: theory and experimental results," IEEE Trans. A utomat. C ontrol, vol. 44, no. 2, pp. 294-309, 1999.

[54] J. S. Thorp, C. E. Seyler, and A. G. Phadke, "E lectromechanical wave propagation in large electric power systems," IE E E Transactions on Circuits and Systems - I: Fundamental Theory and Applications, vol. 45, no. 6, pp. 614-622, 1998.

[55] J. Warga, "Variational problems with unbounded control s," J. SIAM. Ser. A, Control, vol. 3, no. 2, pp. 428-438, 1965.

[56] J. Warga, O ptimal Control of Dixerential and F unctional E quations. New Y ork: A cademic Press, 1972.

[57] J. Warga and Q. J. Zhu, "The equivalence of extremals in dixerent representations of unbounded control problems," SIA M J. Contr. Optim., vol. 32, no. 4,p p. 1151-1169, 1994.

[58] S. T. Zavalishchin and A. N. Sesekin, Dynamic Impulse Systems. Theory and Applications. Dordrecht, The Netherlands: K luwer A cademic P ublishers, 1997. 